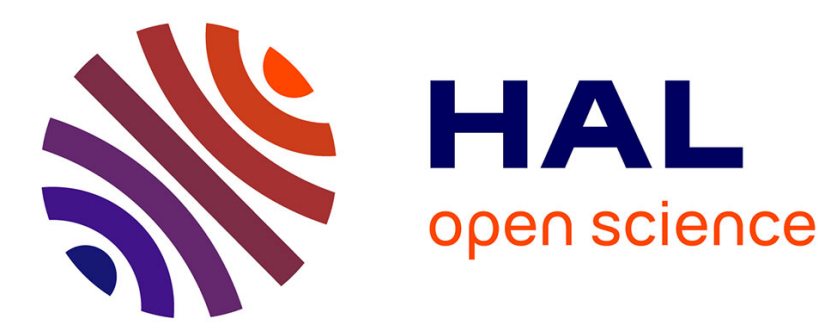

\title{
On some applications of Generalized Geometric Projection to optimal 3D printing
}

Krishnaraj Vilasraj Bhat, Gabriele Capasso, Simone Coniglio, Joseph Morlier, Christian Gogu

\section{- To cite this version:}

Krishnaraj Vilasraj Bhat, Gabriele Capasso, Simone Coniglio, Joseph Morlier, Christian Gogu. On some applications of Generalized Geometric Projection to optimal 3D printing. Computers and Graphics, 2021, 10.1016/j.cag.2021.10.006 . hal-03381445

\section{HAL Id: hal-03381445 \\ https://hal.science/hal-03381445}

Submitted on 16 Oct 2021

HAL is a multi-disciplinary open access archive for the deposit and dissemination of scientific research documents, whether they are published or not. The documents may come from teaching and research institutions in France or abroad, or from public or private research centers.
L'archive ouverte pluridisciplinaire HAL, est destinée au dépôt et à la diffusion de documents scientifiques de niveau recherche, publiés ou non, émanant des établissements d'enseignement et de recherche français ou étrangers, des laboratoires publics ou privés. 


\title{
On some applications of Generalized Geometric Projection to optimal 3D printing
}

\author{
Krishnaraj Vilasraj Bhat ${ }^{\mathrm{a}, \mathrm{b}, 1},{\text { Gabriele } \text { Capasso }^{\mathrm{b}, \mathrm{c}}, \text { Simone Coniglio }^{\mathrm{c}}, \text { Joseph Morlier }^{\mathrm{a}, \mathrm{b}, \mathrm{d}, 1} \text {, Christian Gogu }}^{\mathrm{a}, \mathrm{b}, \mathrm{d}, 1}$ \\ ${ }^{a}$ ISAE SUPAERO, 10 avenue Edouard Belin, 31400 Toulouse, France \\ ${ }^{b}$ Institut Clément Ader, 3 rue Caroline Aigle, 31400 Toulouse, France \\ ${ }^{c}$ Airbus Operations SAS, 316 route de Bayonne, 31100 Toulouse, France \\ ${ }^{d}$ Université Toulouse III - Paul Sabatier, 118 Route de Narbonne, 31062 Toulouse
}

\section{A R T I C L E IN F O}

Article history:

Received October 16, 2021

Keywords: Topology Optimization, Additive Manufacturing Constraints, GGP, ALM, Structural Optimization, Moving Node Method, Overhang angle, Bridge Length

\section{A B S T R A C T}

In recent years, Topology Optimization (TO) gained interest in the scientific community. It assists in finding the best arrangement of material in a design volume. The classical approach named "Solid Isotropic Material with Penalization" (SIMP) associates a fictitious density to each finite element in the domain. While SIMP is described as an implicit approach which can lead to problems with dimensionality of variables, explicit methods adopt a geometric projection of simple elements (eg.: bars) to reduce the number of design variables. This simplifies the geometric interpretation of the optimal architecture. The major explicit methods were recently unified into a general framework, Generalized Geometric Projection (GGP).

Currently it is quite challenging to take into account manufacturing constraints in the topology optimization design phase. Therefore this paper presents an application of the GGP Method to the design of products made by Additive Layer Manufacturing (ALM). Every printed layer constitutes a geometric element, involving design variables relative to position and width. Specific constraints of ALM, including bridge length and overhang angle, can be easily monitored by exploiting the geometric features of the combined elements.

Examples in two dimensions will be reported, analyzing two academic benchmark problems. A comparison to other proven techniques is also detailed. An mean difference of $7.7 \%$ is observed for solutions with only overhang angle constraint, while a mean difference of $11 \%$ is observed for solutions with overhang angle and bridge length constraint. The presented work integrates design and manufacturing, directly identifying the path of the printed layers.

(C) 2021 Elsevier B.V. All rights reserved.

\section{Introduction}

e-mail: k.vilas_raj@yahoo.co.in (Krishnaraj Vilasraj Bhat), gabriele. capassog@gmail .com (Gabriele Capasso), simone. coniglio@airbus . com (Simone Coniglio), joseph.morlier@isae-supaero.fr (Joseph Morlier), christian.gogu@univ-tlse3.fr (Christian Gogu)

${ }^{1}$ Also affiliated to Université de Toulouse, CNRS, INSA, Mines-Albi, Toulouse, France
DDITIVE Layer Manufacturing $(A L M)$ process has gained
increasing interest in research panorama over the past decade. Introduced as mere prototyping, its adoption was extended to a number of application fields [1]. Versatility and 
customization of the products are two of the numerous advantages of such techniques, based on deposition of successive layers. In [2] one can find a complete description of the process, with the relative applications in several industrial fields. Design of supported structures for ALM is straight-forward. However, performance worsening and economic losses arise from support material removal [3]. In particular:

- Support structures typically result in wasted feedstock material as they are not reusable and have to be discarded after removal if not recyclable.

- It implies further manual work and energy in postprocessing.

- Surface finish can be deteriorated after support material removal.

- Printing time increases, as supports are to be printed as well.

Recently, several studies focused essentially on the design of self-supported structures in order to remove the root of the problem. Design of self-supported structures is quite challenging, which can be formulated mathematically in terms of constraints on following parameters [4]:

- angles: typically layers have to be printed with a minimum angle of $45^{\circ}$ with respect to the base;

- overhangs: free-tip overhanging layers are forbidden, since the material could droop and fall, deteriorating the final geometry and undermining performances;

- bridge length: bridges are allowed in some processes, but the maximum length should not overcome a certain limit, in order to avoid permanent damage the inferior layers (due to tension created by self-weight).

In the literature, the reader can find a number of applications of the integration of ALM constraints in the design phase of printable self-supported structures and will be discussed at length in this section.
The past twenty years have seen increasingly rapid advances in the field of structural optimization: in particular, topology optimization is becoming a key figure in the research panorama. This method leads to an optimal structural efficiency, through the removal of unnecessarily placed material, in order to accomplish the minimization of a given objective (such as the compliance of the system).

Since the pioneering work of Bendsoe and Kikuchi (1988) [5], a number of implicit approaches have been proposed, among which: density-based methods (the well-known SIMP algorithm) [6, 7], evolutionary strategies [8] and level-set based methods [9, 10] are pre-eminent. These methods are widely adopted in commercial softwares, such as the well-known $O p$ tistruct. The key feature of such techniques is the parameterization of the topology based on an Eulerian approach, similar to the one adopted in fluid dynamics: every finite element is described locally independently of the surroundings. This leads to an optimization problem with a very large number of design variables (one for each finite element).

In order to reduce the number of design variables and achieve a direct interpretation of the optimal geometry, several explicit methods based on Lagrangian approaches were proposed. These are based on the projection of elementary components onto a fictitious domain, simplifying the generated geometry. Among multiple schemes available in the academic world, there are several prominent ones. Overvelde [11] proposed the Moving Node Approach (MNA), based on the projection of elementary geometric components described via polynomial functions. This is a flow inspired TO approach where the building blocks of the solution are represented by mass nodes. Norato et al. [12] introduced another method, Geometric Projection (GP) to design structures by introducing fixed-width and fixedthickness using continuum-based TO. The signed distance between each element central point and each component surface is computed and is further used to compute the element local volume fraction by means of a spherical sampling window centered in the element centroid. Another approach presented by Zhang et al. [13] focused on an explicit description of the geom- 
etry through beam elements (Moving Morphable Components or MMC) with variable geometry. The geometry is defined using a Topology Description Function (TDF) that has a positive inside the area occupied by the component, zero at the boundary and negative outside the component. These values are used to compute the element stiffness matrix using the ersatz material model. As an extension of MMC, the same group introduced the Moving Morphable Void (MMV) approach [14], which considered the voids as the real components of the structure.

Recently, the major explicit approaches for TO were unified to a general framework, named Generalized Geometric Projection or GGP, by Coniglio et al. [15]. In this work it was demonstrated that all explicit methods differed from one another only in terms of the values of the parameters of the GGP approach. Such framework is described in brief in the next section. Readers should refer to [15] for a detailed explanation and framework setup.

Introduction of established manufacturing constraints into TO always stirred a lot of interest within scientific community. Zhou et al. [16 introduced casting and extrusion constraints into a commercial software and Zuo et al. [17] implemented machining constraints into the SIMP method using a wavelet modified optimizer to improve convergence while Vatanabe et al. [18] has included a wide range of geometrical and manufacturing constraints in a novel projection based TO framework. In [19] and [20] a complete review of methods adopted in the literature to face the issues related to formulation and application of ALM constraints to TO frameworks can be found. This article will, in particular, discuss the obstacles faced during the design of self-supported structures obtained through Fused Deposition Modeling $(F D M)$ where the material is fused and deposited in layers according to the CAD model.

A number of approaches to design ALM enabled structures using implicit TO with density penalization are described in [21] and [22] where projection methods are employed for overhang control and a Heavyside function is used to form the filter for the same. Studies by Langelaar [23, 24] provides a similar concept of using an Additive Manufacturing (AM) filter, which naturally, works better at higher resolution of the design space: however, this works satisfactorily only for overhang angle constraints of $45^{\circ}$. To further develop this method and overcome the limitation in terms of allowed overhang angles, Thore et al. [25] proposed a computationally inexpensive penalty regulation of overhang constraints to prevent stress concentrations at a reduced cost of the objective function, which also results in improved residual strength and fatigue properties. Qian [26] proposes usage of HPI (Heavyside projection based Integral) based undercut and overhang angle control to achieve ALM printable solutions. An alternate method by Leary et al. [27] involves obtaining a SIMP based solution, following which edges are scanned for overhang angle violation and is then post-processed (i.e., addition of structurally functional overhang supports) to obtain printable designs. Mirzendehdel et al. [28] provided an approach where the support structures are constrained to be minimized, rather than employing the overhang constraints.

Other approaches worth noting are hybridized TO schemes where SIMP is used to obtain an intermediate result. Zhang et al. [29] proposed image processing techniques to extract the structural skeleton from an sub-optimized TO run, which can then be explicitly constrained to achieve a specific set of objectives. This methodology can also be extended to recognize overhang angles and undercut areas to obtain AM ready designs. Another innovative method proposed by Mass et al. [30] uses preformed truss-based virtual skeleton to obtain an AM friendly structure, which is then further solved on a continuum based model.

Despite the advancements towards AM friendly solutions, there are numerous drawbacks associated with these methods. For the most part, implicit TO schemes, with or without the AM based constraints or filters are quite slow due to the sheer number of variables involved in an industrial application. Schemes discussed in [21] and other similar methods that simulate the layer by layer method of AM processes are plagued by increased computational times.

Allaire et al. [31] imposed overhang constraints using geometrical members, which also mimicked the layer-by-layer AM 
process and is based on a level-set approach. In this work, selfweight compliance of the intermediate shapes during AM printing process is introduced as a mechanical constraint functional. Such a framework allows to take into account both overhang angle and bridge length constraints. However, these are implicitly defined and, thus, difficult to tune for a specific machine with explicit geometric constraints.

Explicit TO schemes have several attempts at making their solutions ALM compatible. The work of Guo et al. [32] has used both MMC (Moving Morphable Components) and MMV (Moving Morphable Voids), along with angular variation to the design space, or in other words, build orientation, to obtain AM compatible solutions to test problems. Furthermore, Xian et al. [33] proposed a MMC-based methodology, with additional features like minimum build size and penalty methods to impose connectivity between building components. A major contribution from [33] is the determination of the optimal printing plane, together with the optimal structural layout.

On the contrary, Langelaar [34] provided the means to integrate supports, while also providing counter arguments against completely abolishing overhang constraints, in the fact that supports will ultimately be required to perform finishing operations, or will be necessary to provide additional conduits for heat conduction in case of high temperature metal AM processes.

Finally, similar to implicit methods that provide a solution by simulating the layer-wise build characteristic of AM processes [21], we must explore the same scheme for explicit TO, i.e., provide solutions with geometries that closely mimic the AM processes. Therefore a framework is required that provides AM ready solution that accounts for complex geometrical constraints related to ALM processes, which necessitates appropriate geometrical primitives to mimic the printable layers. While methods proposed by Wein et al. [35] and Bhat et al. [36] initially have different inspirations, the solutions converge to have almost the same methodologies that satisfies most of the preliminary requirements. Therefore the framework presented in this article is essentially a superset of the above two meth- ods, where the choice of design variables derives its inspiration from the above stated sources with additional freedom in solution formation to potentially mirror the AM method. This paper presents a methodology to integrate most commonly known ALM geometry constraints into TO design phase, in order to conceive a print-ready structure.

This article is organized as follows. Section 2 discusses the new GGP framework in its formulation, specific method description, geometric primitive selection and its constraints. Section 3 provides the comparative solutions from this framework and Section 4 provides the conclusion for this article.

\section{New framework}

The following section outlines the explicit topology optimization framework at the basis on the problem formulation.

\subsection{Generalized Geometric Projection (GGP) formulation}

The original framework for $G G P$ essentially comprises round ended bars (as shown in Fig 11) as the set of geometric primitives that form the TO solution. Each geometric primitive has a set of 5 variables (geometric position $x$ and $y$, length $l$, breadth $b$ and angle $\theta$ ), and a characteristic function $\Upsilon$, which defines the density of the set of points inside the primitive, given by:

$$
\Upsilon\left(\left\{X_{g}\right\}, \omega_{i}\right)= \begin{cases}1 & \text { if }\left\{X_{g}\right\} \in \omega_{i} \\ 0 & \text { otherwise }\end{cases}
$$

Where $\left\{X_{g}\right\}$ is the design domain and $\omega_{i}$ is the geometric primitive. It must be acknowledged that the geometric primitives do not define this framework, but rather the problem formulation which potentially gives rise to the three methods that will be explained in the following sections. These geometrical primitives are used to update a fixed mesh finite element model by projection and boolean addition methods. A local density formulation, considered as an estimation using weighted volume fraction is used for the projection, given by:

$$
\begin{aligned}
\delta_{i}^{e l} & =\frac{\int_{D\left(\left\{X_{g}^{e l}\right\}, p, R\right)} W_{i}\left(\{X\},\left\{X_{i}\right\},\{r\}\right) d \Omega}{\int_{D\left(\left\{X_{g}^{e l}\right\}, p, R\right)} d \Omega} \\
& \approx \frac{\sum_{k=1}^{N_{g p}} \varphi_{k} W_{i k}}{\sum_{k=1}^{N_{g p}} \varphi_{k}}
\end{aligned}
$$




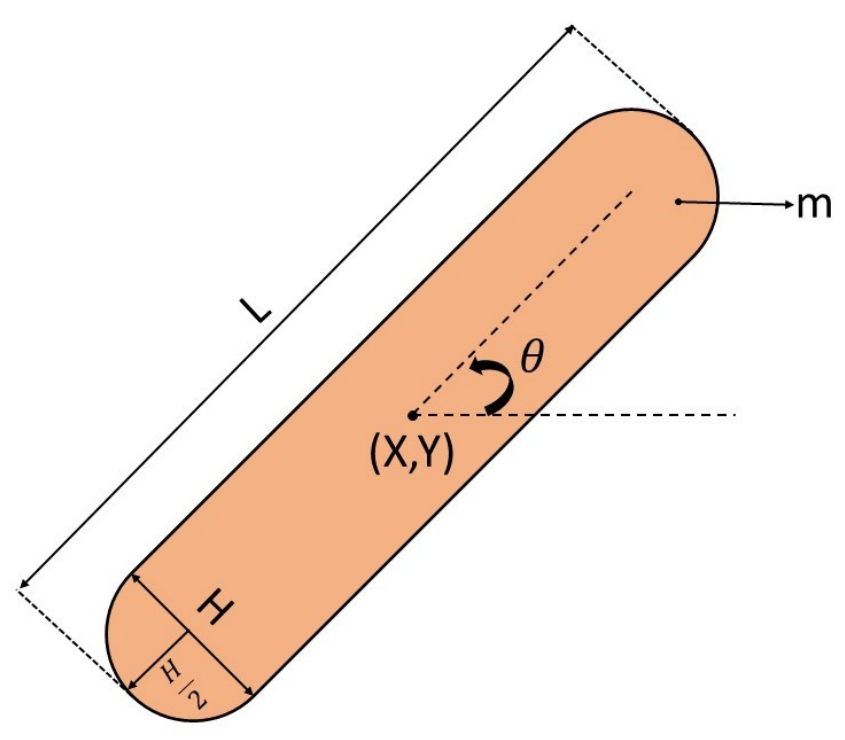

Fig. 1: Example component schematic.

Where $D\left(\left\{X_{g}^{e l}\right\}, p, R\right)$ is the design domain or sampling window, $W_{i}\left(\{X\},\left\{X_{i}\right\},\{r\}\right)$ is a regular continuous approximation of $\Upsilon\left(\left\{X_{g}\right\}, \omega_{i}\right),\{r\}$ is a vector of hyper parameters, $W_{i k}$ is equal to the characteristic function value at Gauss point location, $p$ is the p-norm used for the definition of the sampling window, $N_{g p}$ is the number of Gauss points over which integration is performed, $R$ is the radius of the sampling window and $\varphi_{k}$ are integration weights for the Gauss points. Using the notation of $\left\{\delta^{e l}\right\}_{v}$ and $\left\{\delta^{e l}\right\}_{c}$, described as the vector of local volume fractions computed using density characteristic functions $W^{v}$ and $W^{c}$ respectively in the $e l^{\text {th }}$ element centroid, the final step involves updating the fixed mesh of the FE model using the equation

$$
\begin{gathered}
E^{e l}=\mathbb{M}\left(\left\{\delta^{e l}\right\}_{c}, E, E_{\text {min }}, \kappa\right) \\
\rho^{e l}=\mathbb{V}\left(\left\{\delta^{e l}\right\}_{c}, \kappa\right)
\end{gathered}
$$

Where $\mathbb{M}$ and $\mathbb{V}$ are functions that provide the Young's' Modulus and the densities of each finite element as the resultant of being within a geometric primitive boundary, $E$ is the Young's modulus of the material, $E_{\text {min }}$ is the Young's modulus of a very soft material used to prevent stiffness matrix singularity and illconditioning and $\kappa$ is the aggregation constant chosen for a specific aggregation method.
Next, the method of calculation of Youngs' modulus and element density depends on the specific choices of the parameters $p, N_{g p}, R$ and $W$. It will be demonstrated that the previously discussed method of MMC, GP and MNA are specific cases of the GGP framework.

\subsubsection{Adapted Moving Morphable Components (AMMC)}

The original MMC framework employs a regularized Heavyside function $H(x)$ applied to the TDF $\chi$ of the union of all components. For the AMMC framework, $W^{v}=H(\chi), W^{c}=H(\chi)^{q}$, with a sampling window radius $R$ resulting in a sampling window of area $\mathbf{D}$ and $N_{g p}$ Gauss points, on a 2D design space are considered. The following expression is considered for providing an elements Youngs' Modulus:

$$
\mathbb{M}\left(\left\{\delta^{e l}\right\}_{c}, E, E_{\text {min }},\right)=\lim _{p} \delta^{e l}\left(W^{c}, p, R\right) E_{0}
$$

with $E_{0}$ being the material Youngs' modulus, analogous to $E$. Therefore the volume covered by a sampling window (denoted as the denominator in Exp. 2) is given by:

$$
\lim _{p} \int_{D\left(\left\{X_{g}\right\}, p, R\right)} d \Omega=\sum_{j=1}^{N_{g p}} D
$$

Therefore the local volume fraction shown by Exp. 2 is now:

$$
\lim _{p} \delta^{e l}\left(W^{c}, p, R\right) \approx \frac{D \sum_{j=1}^{N_{g p}}\left(H\left(\chi\left(x_{j}\right)\right)\right)^{q}}{\sum_{j=1}^{N_{g p}} D}
$$

and the Youngs' modulus is described as:

$\mathbb{M}\left(\delta^{e l}\left(W^{c}, p, R\right), \kappa, E_{0}\right)=\lim _{p} \delta^{e l}\left(W^{v}, p, R\right) E_{0} \approx E_{0} \frac{\sum_{j=1}^{N_{g p}} H\left(\chi\left(x_{j}\right)\right)^{q} D}{\sum_{j=1}^{N_{g p}} D}$

Similarly, the density is now expressed as:

$$
\mathbb{V}\left(\delta^{e l}\left(W^{v}, p, R\right), \kappa\right)=\lim _{p} \delta^{e l}\left(W^{v}, p, R\right) \approx \frac{\sum_{j=1}^{N_{g p}} H\left(\chi\left(x_{j}\right)\right) D}{\sum_{j=1}^{N_{g p}} D}
$$

These formulations for element density and Youngs' Modulus provides the basic framework for AMMC. Using the values $p \rightarrow \infty, R=\frac{\sqrt{3}}{2} d x$ and $N_{g p}=4$ resulting in a square sampling window of area $R^{2}$, one can recalculate the $\operatorname{Exp} 6,7,8$ and 9 as: 


$$
\begin{gathered}
\lim _{p \rightarrow \infty} \int_{D\left(\left\{X_{g}\right\}, p, R\right)} d \Omega=3 d x^{2} \\
\lim _{p \rightarrow \infty} \delta^{e l}\left(W^{c}, p, R\right) \approx \frac{\sum_{j=1}^{N_{g p}}\left(H\left(\chi\left(x_{j}\right)\right)\right)^{q}}{4} \\
\mathbb{M}\left(\delta^{e l}\left(W^{c}, p, R\right), \kappa, E_{0}\right) \approx E_{0} \frac{\sum_{j=1}^{N_{g p}} H\left(\chi\left(x_{j}\right)\right)^{q}}{4} \\
\mathbb{V}\left(\delta^{e l}\left(W^{v}, p, R\right), \kappa\right) \approx \frac{\sum_{j=1}^{N_{g p}} H\left(\chi\left(x_{j}\right)\right)}{4}
\end{gathered}
$$

respectively, which provides the original MMC framework. It must be stated that the hyperparameters can be tuned to provide better convergence during optimization.

\subsubsection{Adapted Geometric Projection (AGP)}

The AGP framework uses a sampling window of radius $R$ that results in a sampling window of area $\mathbf{D}$, and $N_{g p}$ Gauss points and replaces $W_{i}$ with $\Upsilon_{i}$ or $\delta_{i}^{e l}$ in Exp. 2 depending on the hyperparameters chosen (refer to [15] Section 2.4), with the assumption that the boundary $\left(\partial \omega_{i}\right)$ of a given component $(\omega)$ is restricted to be straight ([37], [38]). The parameter $p$ remains a variable.

The local volume fraction at the $e l^{t h}$ element composed by $n$ Gauss points by $i^{t h}$ component is directly computed without using any weighting function, given by:

$$
\lim _{p} \delta^{e l}(\Upsilon, p, R)=\frac{\sum_{j=1}^{N_{g p}} \Upsilon_{i n}^{e l} \varphi_{n}}{\sum_{j=1}^{N_{g p}} \varphi_{n}}
$$

In order to prevent stiffness matrix singularities during computation, the volume fraction is modified to have a minimum value $\delta_{\min }$, given by:

$$
\tilde{\delta}^{e l}=\delta_{\min }+\left(1-\delta_{\min }\right) \delta_{i}^{e l}
$$

Therefore, the resulting local density per element is given by:

$$
\rho^{e l}=\mathbb{V}\left(\{\delta\}^{e l}, \kappa\right)=\Pi\left(\left\{\hat{\delta}^{e l}\left(r, \gamma_{v}\right)\right\}, \kappa\right)
$$

$$
\text { where } \hat{\delta}^{e l}=\tilde{\delta}_{i}^{e l} m_{i}^{\gamma}
$$

And for the local Young's Modulus, we have:

$$
E^{e l}=\mathbb{M}\left(\{\delta\}^{e l}, E, E_{\min } \kappa\right)=\Pi\left(\left\{\hat{\delta}^{e l}\left(r, \gamma_{c}\right)\right\}, \kappa\right) E
$$

Where $m_{i}$ is the mass value of the $i^{t h}$ component, $\gamma$ is the penalty factor and $\Pi(x)$ is a smoothed approximation of maximum function. The above formulae represents the AGP framework. Considering $p \rightarrow \infty, R=\frac{1}{2} d x$ and $N_{g p}=1$, we obtain a circular sampling window of area $\mathbf{D}=\mathbf{B}_{P}^{r}$, where $\left|\mathbf{B}_{P}^{r}\right|=\pi r^{2}$, and with $W_{i}^{e l}=\delta_{i}^{e l}$, Exp 14 can be recalculated as:

$$
\delta_{i}^{e l}=\frac{\left|\mathbf{B}_{P}^{r} \cap \omega_{i}\right|}{\left|\mathbf{B}_{P}^{r}\right|}
$$

The above expression recovers the original GP framework [37], which signifies that the AGP framework is a superset of the GP method. It must be noted that the hyperparameters can be varied to suit the problem at hand or improve convergence.

\subsubsection{Adapted Moving Node Approach (AMNA)}

As briefly discussed in Section 1, Overvelde [11] proposed a flow-inspired topology optimization approach called the MNA, where the solution is formed using mass nodes. The weighting functions are directly applied to these values to compute the local density contributed by each mass node. The densities from each contributing node is summed and an adapted procedure called the asymptotic density is used to prevent the density obtain a value greater than 1 . The MNA method works using both meshless and FEA methods for displacement evaluation, with a view to reduce the number of variables and degrees of freedom.

The AMNA framework is different than the proposed MNA method, in that instead of using mass nodes, 2D geometric primitives with clear boundaries are used in a FEA based implementation. To formulate the AMNA framework, $W_{i}=$ $m_{i}^{\gamma} \omega\left(v_{i}, h_{i}, \varepsilon_{i}\right)$ must be used in $\operatorname{Exp} 2$. The hyperparameters $p$, $R$ and $N_{g p}$ are once again variables. Therefore $\operatorname{Exp} 2$ now is:

$$
\lim _{p} \delta_{i}^{e l}\left(\omega_{i}, p, R\right)=\frac{\sum_{j=1}^{N_{g p}} m_{i}^{\gamma} \omega_{i n}^{e l} \varphi_{n}}{\sum_{j=1}^{N_{g p}} \varphi_{n}}
$$

Where $\omega_{i n}^{e l}$ is the weighting function of the component $i$ at the $n_{t h}$ gauss point at the $e l_{t h}$ element. In the AMNA framework, the local densities are directly the volume fractions (therefore $\delta=$ $\rho$, subscripts and superscripts omitted for brevity), and therefore 
the local densities and Youngs' modulus, with $p_{b}$ described as the AMNA penalty factor, are expressed as:

$$
\begin{gathered}
\mathbb{V}\left(\{\delta\}^{e l}, \kappa\right)=\Pi\left(\{\delta\}_{v}^{e l}, \kappa\right) \\
\mathbb{M}\left(\{\delta\}^{e l}, \kappa\right)=E_{\text {min }}+\left(E-E_{\text {min }}\right) \Pi\left(\{\delta\}_{c}^{e l}, \kappa\right)^{p_{b}}
\end{gathered}
$$

Where in this case, $\Pi()$ refers to the asymptotic density function used. Using the values $p \rightarrow \infty, R=\frac{1}{2}$ with $N_{g p}=1$, $\operatorname{Exp} 20$ is now recalculated as:

$$
\delta_{i}^{e l}=m_{i}^{\gamma} \omega_{i}^{e l}
$$

It can be observed that the Exp 20 reflects the original MNA formulation. Therefore, the MNA is effectively a subset of the AMNA framework. The hyperparameters can again be varied to suit the problem at hand.
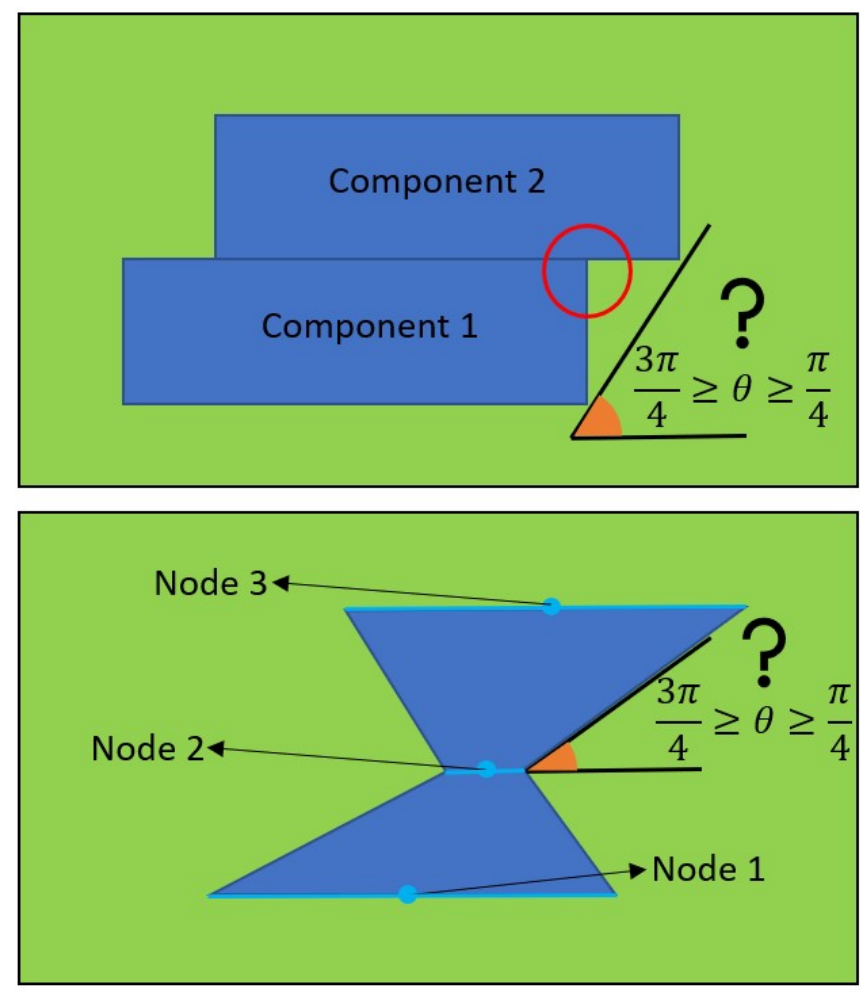

Fig. 2: A simple comparison of 2D components against 1D node-based components in view of overhang angle violation. [Top] A schematic of 2D components under influence of the overhang constraint. The red circle demarcates the area where the angle constraint is almost always violated. [Bottom] A schematic of the $1 \mathrm{D}$ based component system that always adheres to the overhang constraints.

\subsection{Geometric Primitive Selection}

Contrary to the previous section that stated round ended bars as the geometric primitives, the variables chosen for the explicit geometry consist of 2 variables per geometry, denoted by $x_{k}$ (lateral position of $k^{\text {th }}$ component center) and $l_{k}$ (length of $k^{\text {th }}$ component), effectively reducing the original 2 dimensional primitive into 1 dimensional geometric primitive. Another method to describe the geometric primitive is to consider it as a lofted 2D geometry, i.e., a 2D shape consisting of multiple $1 \mathrm{D}$ lines (or called a component in this context) connected by 2 variably curved lines. Each $1 \mathrm{D}$ lines have a node (i.e., a centrepoint) and a length as its variables. In analogy with ALM technology, this corresponds to the evolution in spacetime of the printing process itself. It must be noted that the node does not have the freedom to move longitudinally to better suit the ALM processes, and hence, the variable $y$ will not be taken into consideration. From a given component, another component is placed after ' $n$ ' spaces above it, and is continued until the boundary of the design space is reached. This results in a 'band' like structure (previously described as the lofted 2D geometry) that is free to stretch, shrink and displace itself around the design space. Further, each 'band' of nodes have density $m$ and print height $h$ to enable the optimizer to include only the relevant components (or a part of it) in the final solution. Such a variable choice also has an additional advantage that is quite important in applying the AM constraint, i.e., the ability of the resulting geometry to have a gradual overhang angle evolution, rather than a step-based evolution of the same, which from Fig. 2, implies that the overhang constraint is almost always violated. For added flexibility towards the range of solutions formed, the design space orientation $\theta_{0}$ and rotation elevation $y_{0}$ are also variables to be optimized, as using a strict south to north approach to $3 \mathrm{D}$ printing is not always beneficial. This primitive seems to introduce a higher number of variables compared to the original GGP framework. An important aspect to consider is the possibility of having a higher resolution of the design space. In such cases, the presented frameworks' total number of variables remain invariable, while traditional implicit 
methods like SIMP introduce very large number of variables.

By fixing the number of components $n_{p}$ and the number of layers $n_{y}$, the design variables are now summarized as:

- $x_{i j}$ the midpoint abscissa of the segment $j$ of the $i$-th component (with $j=0, \ldots, n_{y}+1, i=1, \ldots, n_{p}$ );

- $l_{i j}$ the length of the segment $j$ of the $i$-th component (with $\left.j=0, \ldots, n_{y}+1, i=1, \ldots, n_{p}\right)$;

- $m_{i}$ the mass variable of component $i\left(i=1, \ldots, n_{p}\right)$;

- $h_{i}$ the printing height variable of component $i$ ( $i=$ $\left.1, \ldots, n_{p}\right)$

- $y_{0}$ the translation of the printing plan;

- $\theta_{0}$ the rotation of the printing plan, as represented in Fig. 3.

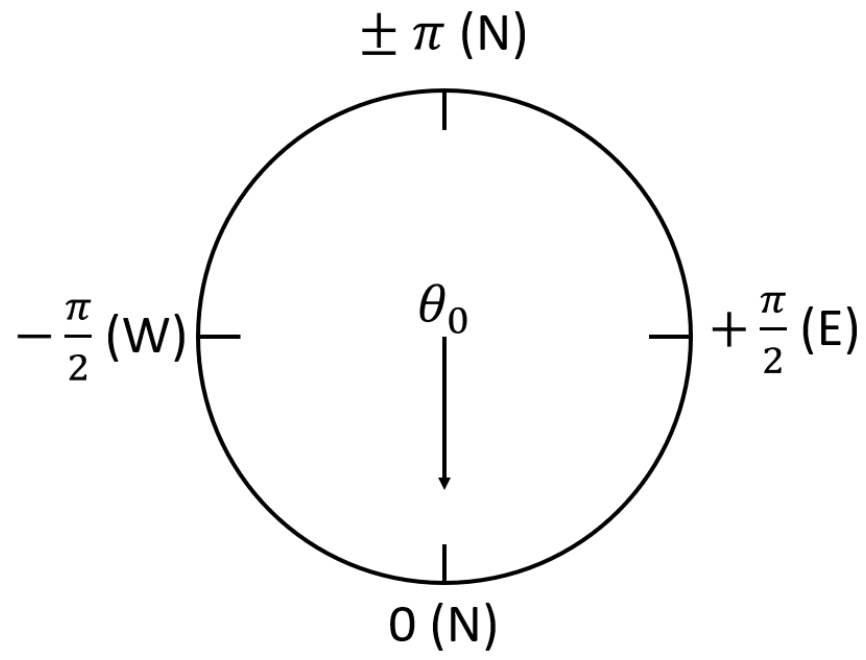

Fig. 3: A pictorial representation of the continuously varying print angle, along with the baseplate system adopted in SIMP+AMfilter framework.

In this framework, $m_{i}$ definition is analogue to the variable introduced by Norato [37]: thanks to this parameter, it is possible to remove a component from the solution. Moreover, the possibility of printing only a portion of the entire component $i$ is allowed by the height variable $h_{i}$ : this represents the ratio between the height of the portion of the component which should actually be printed and the maximum (characteristic) length of the segment which is included in the design space. As a final remark, it should be noted that translation and rotation are applied on the design space itself and not on geometrical components space. A pictorial representation of the components and the variables is provided in Fig. 4 for clarity.

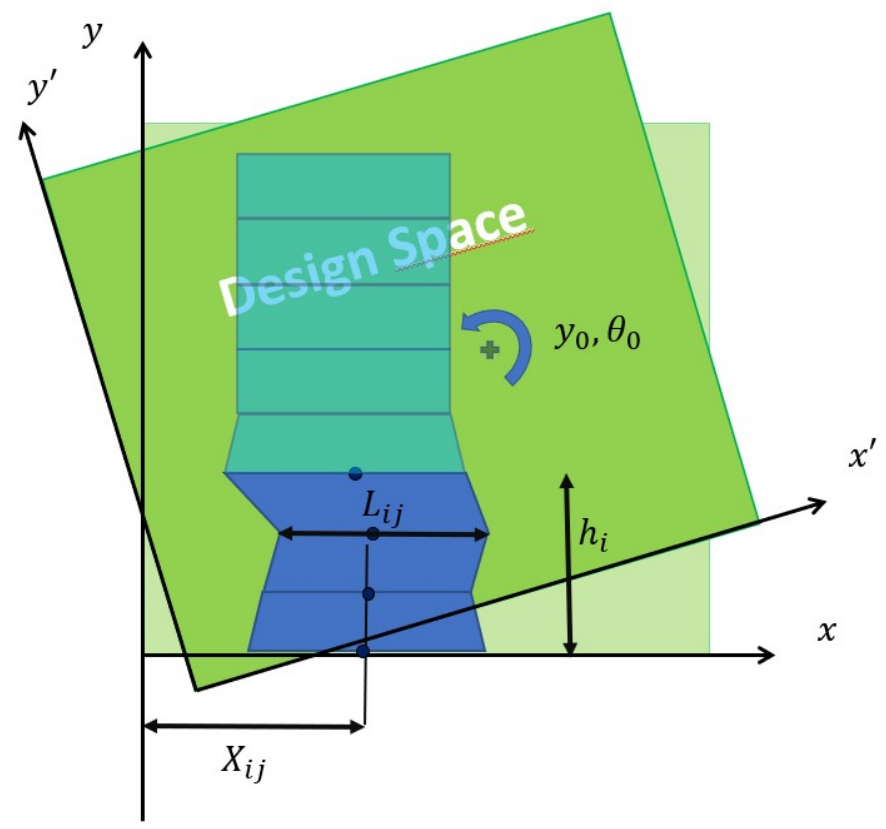

Fig. 4: A representation of all the framework variables within the design space. The variables $X$ and $L$ denote the lateral control point and area of influence, while $m, h, y_{0}$ and $\theta_{0}$ denote the component mass, print height (printed height shown in dark blue), rotation elevation and design space orientation respectively.

\subsection{Specific Framework Formulation}

After having dealt with the methods used within the GGP framework, the exact formulation used for the optimization process will be discussed below. To provide a definite set of expressions, the MMC, GP and MNA subcases of AMMC, AGP and AMNA will be used.

The objective function in this problem statement will be limited to the most commonly used objectives for structural TO problems, i.e., to minimize the compliance of the final solution, given by:

$$
\left\{\begin{array}{l}
\min _{\{x\}} C=\{U\}^{T}\{F\} \\
\text { s.t } \\
V=\frac{\sum_{e l=1}^{N} \rho^{e l}}{N} \leq V_{0} \\
\left\{l_{b}\right\} \leq\{x\} \leq\left\{u_{b}\right\}
\end{array}\right.
$$

Where $\{x\}$ is the design vector to be determined, $\left\{l_{b}\right\}$ and $\left\{u_{b}\right\}$ is its lower and upper bound respectively, $\{F\}$ is the external imposed loads on the design space, $C$ is the compliance of the 
resulting structure, $\{U\}$ is a vector of nodal displacements, $V_{0}$ is the maximum volume fraction allowed, $N$ is the total number of elements and $\rho^{e l}$ is the density of a given element in the design space. The displacement $U$ is obtained using the static force balance equation given by:

$$
\begin{gathered}
\qquad K]\{U\}=\{F\} \\
\text { and therefore }\{U\}=[K]^{-1}\{F\}
\end{gathered}
$$

Where $[K]$ is the assembled global stiffness matrix. The global design space is discretized into a $N$ number of solid elements with unit thickness, over which the geometric primitives are arranged and project their topology. The finite element theory dictates:

$$
[K]=\bigoplus_{e l=1}^{N}\left[K^{e l}\right]
$$

$$
\text { where }\left[K^{e l}\right]=E^{e l}\left[K_{0}\right]
$$

Where $\left[K^{e l}\right]$ is the elementary stiffness matrix, $\left[K_{0}\right]$ is the 8 $\times 8$ stiffness matrix of a $d x \times d x$ plane stressed solid element, and $[K]$ is the assembled global stiffness matrix. It must be highlighted that $[K]$ must be non singular matrix, and therefore future formulations that lead to the stiffness matrix assembly will be taken into consideration. The geometric primitives (and hence the design variables) were discussed in the previous section.

To avoid the singularity of $[K]$ matrix, which is dependent on the density $\rho^{e l}$, the notation provided by Bendsoe and Kikuchi in the SIMP methodology [5] is used:

$$
E^{e l}=E_{\text {min }}+\left(E-E_{\text {min }}\right) \rho^{p}
$$

Where $E^{e l}$ is the element Young's modulus, $E_{\min }$ is a minimum sufficient to avoid singularities and $\rho$ is the density penalized using the penalty $p$. This ensures that the design obtained is roughly $0 / 1$ density field, rather than consisting of a broad range of intermediate densities in $[0,1]$. It is now necessary to investigate methods to obtain the value of density $\rho$, the expressions of which is dependent on the method chosen in the framework, and is discussed in the next sub-sections.

The region of influence due to the projected geometries at a vertical height $y$ in between two successively stacked nodes, whose lateral, longitudinal position and length are given by $\left(x_{1}, x_{2}\right),\left(y_{1}, y_{2}\right)$ and $\left(l_{1}, l_{2}\right)$ respectively, is calculated using simple geometry. Consider a point $\left(l_{x}, u_{x}\right)$ that represents the extremes of the influenced region. The equation is given by:

$$
\begin{aligned}
& l_{x}=\left(x_{1}-\frac{l_{1}}{2}\right)+\left(\frac{y-y_{1}}{y_{2}-y_{1}}\right)\left(x_{2}-\frac{l_{2}}{2}-x_{1}+\frac{l_{1}}{2}\right) \\
& u_{x}=\left(x_{1}+\frac{l_{1}}{2}\right)+\left(\frac{y-y_{1}}{y_{2}-y_{1}}\right)\left(x_{2}+\frac{l_{2}}{2}-x_{1}-\frac{l_{1}}{2}\right)
\end{aligned}
$$

Whereas, $\left(l_{y}, u_{y}\right)$ represents the longitudinal position of the nodes below and above a given Gauss point. For every component we introduce the height which should be printed. For a rectangular design space, we have:

$$
b=\sqrt{N_{e l x}^{2}+N_{e l y}^{2}} * h
$$

where $N_{\text {elx }}$ and $N_{\text {ely }}$ are the number of elements of the design space along the $x$ and $y$ axes respectively, and $\mathrm{h}$ is the print height fraction for the specified component. It is formulated in the given manner in order to enable the component to stretch to the maximum dimension possible for the given design space, i.e., the diagonal. Since the design space orientation is a variable, the Gauss points must also be changed to reflect the translation and rotation of the design space. The related equations are given by:

$$
\begin{gathered}
x_{g}=\frac{N_{e l x}}{2}+\left(x-\frac{N_{e l x}}{2}\right) \cos \theta_{0}+\left(y-\frac{N_{e l y}}{2}\right) \sin \theta_{0} \\
y_{g}=\frac{N_{e l y}}{2}-y_{0}-\left(x-\frac{N_{e l x}}{2}\right) \sin \theta_{0}+\left(y-\frac{N_{e l y}}{2}\right) \cos \theta_{0}
\end{gathered}
$$

The variable $y_{0}$, while not always required for the complete solution, is meant to achieve several objectives. One being that $y_{0}$ helps achieve complete connectivity in case of change in design space orientation for an uneven design space, while offering more stability during optimization. Hence, the variables 
to optimize are component lateral position and length $(x \& l)$ (Eq. 30 and 31, component mass $m$ (Eq. 23, component print height $h\left(\mathrm{Eq} 32\right.$ and $\mathrm{Eq} 37 \mathrm{p}$, design space orientation $\theta_{0}$ and longitudinal displacement $y_{0}(\mathrm{Eq} 33$ and $\mathrm{Eq} 34$.

Having known the region of influence over the whole design space, instead of just near the nodes, the characteristic function value $W$ is given by the method chosen in the framework, i.e., AMMC, AMNA or AGP.

\subsection{1. $A M M C$}

The AMMC method requires the usage of Topological Description Function (TDF) to obtain the structural topology. With the given region of influence outlined by $l_{x}, u_{x}$, the TDF function is given by:

$$
\chi=1-\left(\frac{4\left(x_{g}-\frac{u_{x}+l_{x}}{2}\right)^{2}}{\left(u_{x}-l_{x}\right)^{2}}\right)^{\alpha}-\left(\frac{y_{g}^{2}}{b^{2}}\right)^{2 \alpha}
$$

Subsequently, the presence or absence of material due to the primitives is established by using a regularized (smoothed) Heavyside function given by:

$$
H_{\epsilon}(\chi)= \begin{cases}1 & \text { if } \chi>\epsilon \\ \frac{3(1-\beta)}{4}\left(\frac{\chi}{\epsilon}-\frac{\chi^{3}}{3 \epsilon^{3}}\right) & \text { if }-\epsilon \leq \chi \leq \epsilon, \\ \beta & \text { otherwise. }\end{cases}
$$

where the amplitude of translation between the minimum value $\beta$ and 1 is controlled by the parameter $\epsilon$. Subsequently, Eq 9 is used to obtain the local density over the design space.

\subsection{2. $A G P$}

The AGP method relies on using signed distances from each Gauss point to each of the nodal extremities. $\zeta_{1}$ and $\zeta_{2}$ are specifically the shortest distance of each Gaussian point from the LHS and RHS boundaries of each component band as shown in Fig. 5. Consequently the equations are given by:

$$
\zeta= \begin{cases}\left(l_{x}-x_{g}\right) * \sin \theta_{l} & =\zeta_{1} \\ \left(x_{g}-u_{x}\right) * \sin \theta_{r} & =\zeta_{2} \\ \left(y_{g}-b\right) & =\zeta_{3} \\ \left(l_{y}-y_{g}\right) & =\zeta_{4} \\ \left(y_{g}-u_{y}\right) & =\zeta_{5}\end{cases}
$$

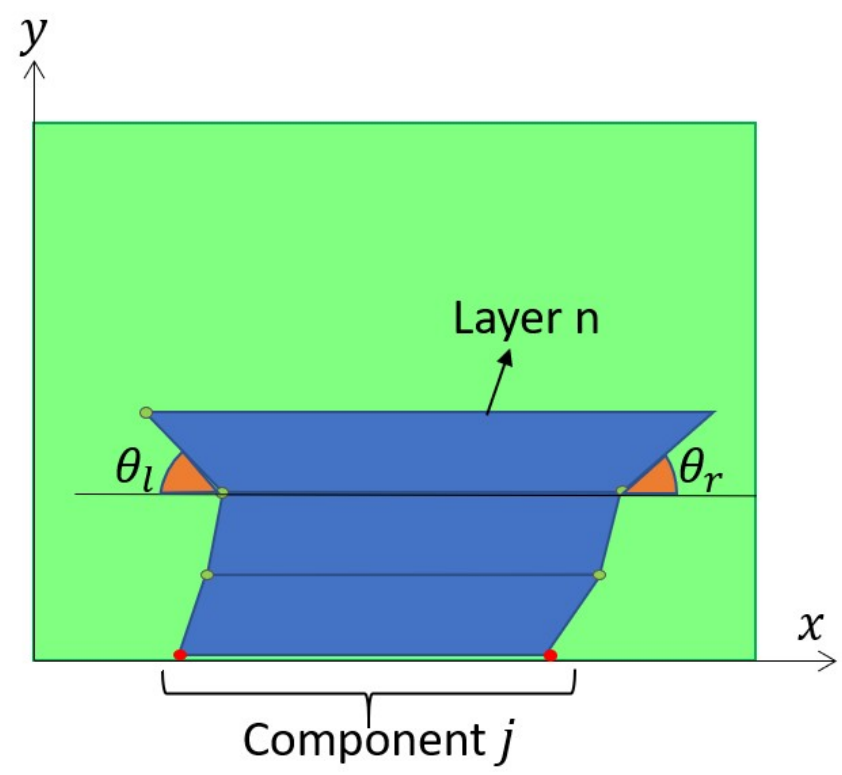

Fig. 5: A simple schematic that displays the component angles $\theta_{l}$ (for LHS) and $\theta_{r}$ (for RHS) between layers $n$ and $n-1$ of a component $\mathrm{J}$.

Using the hyperparameters given in Section 2.1.2 for the GP subset of AGP, the expression 19 is now:

$$
\delta_{i}^{e l}= \begin{cases}0 & \text { if } \zeta>r, \\ \frac{1}{\pi r^{2}}\left[r^{2} \arccos \frac{\zeta}{r}-\zeta \sqrt{r^{2}-\zeta^{2}}\right] & \text { if }-r \leq \zeta \leq r, \\ 1 & \text { otherwise. }\end{cases}
$$

The equations in Eqs. $16 \& 18$ further state that in case of overlapping density values due to presence of multiple components, a maximizing function considered across $\delta_{i}^{e l}\left(\zeta_{1}\right)$ to $\delta_{i}^{e l}\left(\zeta_{5}\right)$ is used to calculate the final density and Youngs modulus respectively at a given Gauss point.

\subsubsection{AMNA}

For clarity and complete set of formulations, the variables assumed in Section 2.1.3 to derive the original MNA framework from AMNA will be used. We introduce five test variables, which are meant to express whether a Gauss point is inside or outside the boundary of the layer of a component and below the maximum printed height $b$. In particular, we have:

$$
\zeta_{i}=\left\{\begin{array}{l}
l_{x}-x_{g} \\
x_{g}-u_{x} \\
y_{g}-b \\
l_{y}-y_{g} \\
y_{g}-u_{y}
\end{array}\right.
$$

In particular, for a given Gauss point, having all $\zeta \leq 0$ implies that the point itself lies inside the boundaries of a prescribed 
layer of a component. The equation for the weighting function $W$, with $\zeta$ being the distance from the element centroids to the component edges is given by:

$$
W\left(\zeta_{i}\right)=0.5-\left(\frac{15}{16 \sigma}\right) \zeta_{i}+\left(\frac{5}{8 \sigma^{3}}\right) \zeta_{i}^{3}-\left(\frac{3}{16 \sigma^{5}}\right) \zeta_{i}^{5}
$$

and therefore, the elemental densities are given by:

$$
\rho_{i}^{e l}=\prod_{i=1}^{5} W\left(\zeta_{i}\right)
$$

Further, in order to perform the summation of densities due to all mass nodes, the asymptotic density function is employed as shown in Eq. 21 .

\subsection{ALM constraints}

Described in detail in section 2.2, the geometric primitives themselves have been selected to suit the constraints ALM processes requires. While there are quite a lot of parameters to optimize for an AM enabled solution, this article will solely deploy the overhang angle and bridge length constraints. As mentioned before, the optimum minimum overhang angle that must be adhered to prevent drop off or other defects is set at 45 degrees on either sides, and the geometric primitives adapted for this formulation makes it quite simple in its implementation. A tabulated reference is provided below in Table 1 for an overview of the self supported additive manufacturing constraints tackled in open domain until now, detailed in Section 1 .

Following the notation provided in equations 30 . 32 for two successively stacked nodes, the overhang angle on either side of the nodes is obtained by a simple geometric equation as follows:

$$
\begin{aligned}
& \tan \alpha=\frac{\left(x_{2}+\frac{l_{2}}{2}\right)-\left(x_{1}+\frac{l_{1}}{2}\right)}{\frac{N_{e l y}}{n_{y}-1}} \\
& \tan \beta=\frac{\left(x_{1}-\frac{l_{1}}{2}\right)-\left(x_{2}-\frac{l_{2}}{2}\right)}{\frac{N_{e l y}}{n_{y}-1}}
\end{aligned}
$$

Where $n_{p}$ and $n_{y}$ are the total number of component layers (and hence their centers) stacked horizontally and vertically. Therefore, the formulation for the constraints is now fairly straightforward, given by

$$
C_{1}=\frac{\tan \alpha-\tan (45)}{\tan (45)}
$$

$$
C_{2}=\frac{\tan \beta-\tan (45)}{\tan (45)}
$$

The next constraint to be implemented is the bridge length constraint (represented in Fig 6) that is realistically dependent on various factors like material to be deposited, the printing method employed and so on. The bridge length here represents the maximum distance a layer of a component can be printed on both sides with respect to the edges of the base layer. Results provided below use arbitrary bridge length limits to represent the efficacy of the framework and is independent of any known values. The equation for this constraint is given by:

$$
\begin{aligned}
& C_{3}=\Delta U_{i j}=\left|u_{i j}-u_{0 j}\right| \leq \frac{B L_{\text {max }}}{2} \\
& C_{4}=\Delta L_{i j}=\left|l_{i j}-l_{0 j}\right| \leq \frac{B L_{\text {max }}}{2}
\end{aligned}
$$

Where $u_{i j} \& l_{i j}$ represents the right and left endpoint abscissa of the segment $i$ of component $j$ respectively, while $B L_{\text {max }}$ is the maximum bridge length constraint to be imposed. The main idea is to restrain the variability of the position of every endpoint to a window centered around the endpoint of the base segment $(i=0)$ : right endpoints are constrained with respect to the right endpoint of the base segment, and conversely for the left endpoints. This constitutes a conservative measure of bridge length, as it ensures that the maximum distance between two segments endpoints do not exceed the value imposed by $B L_{\max }$. A less conservative measure could be given by the actual distance of every combination of segments at the same ordinate level from all components: as this implies a far superior number of constraints, the authors opted for the first solution.

\subsection{Constraint Aggregation}

Scrutinizing the constraints provided in section 2.4 , it can be concluded that the number of constraints across $n_{p} \times n_{p}$ component centers are quite large, precisely, $2 * n_{p} *\left(n_{y}-1\right)$ for any selected AM constraint. This presents a real dilemma, as 


\begin{tabular}{|c|c|c|c|c|c|}
\hline References & Check On & $\begin{array}{c}\text { Overhang } \\
\text { Angle }\end{array}$ & $\begin{array}{c}\text { Bridge } \\
\text { Length }\end{array}$ & $\begin{array}{c}\text { Optimal } \\
\text { Printing } \\
\text { Plane }\end{array}$ & Comment \\
\hline SIMP [27] & Boundaries & Yes & No & No & Additional Iterations \\
\hline $\begin{array}{c}\text { SIMP + AM Filter } \\
{[24]}\end{array}$ & Densities & Yes & No & No & One constraint per element \\
\hline Level Set [31] & Boundaries & Yes & Yes & No & Implicit Constraints \\
\hline MMC \& MMV [32] & Boundaries & Yes & No & No & \\
\hline MMC [33] & $\begin{array}{c}\text { Component } \\
\text { Angles }\end{array}$ & Yes & No & Yes & Difficult Quality Check \\
\hline
\end{tabular}

Table 1: Tabulated current approaches to topology optimization for self supported additive manufacturing

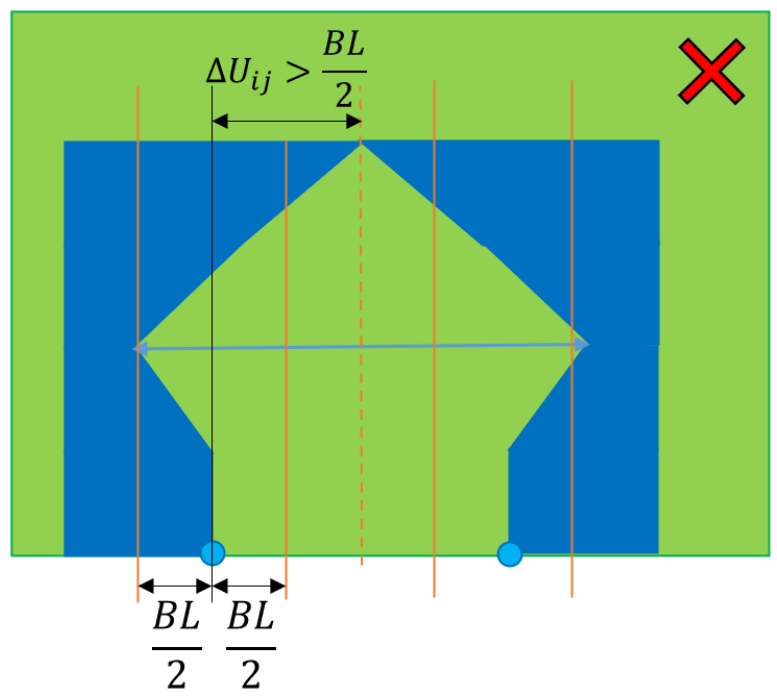

(a) Bridge length constraint violated

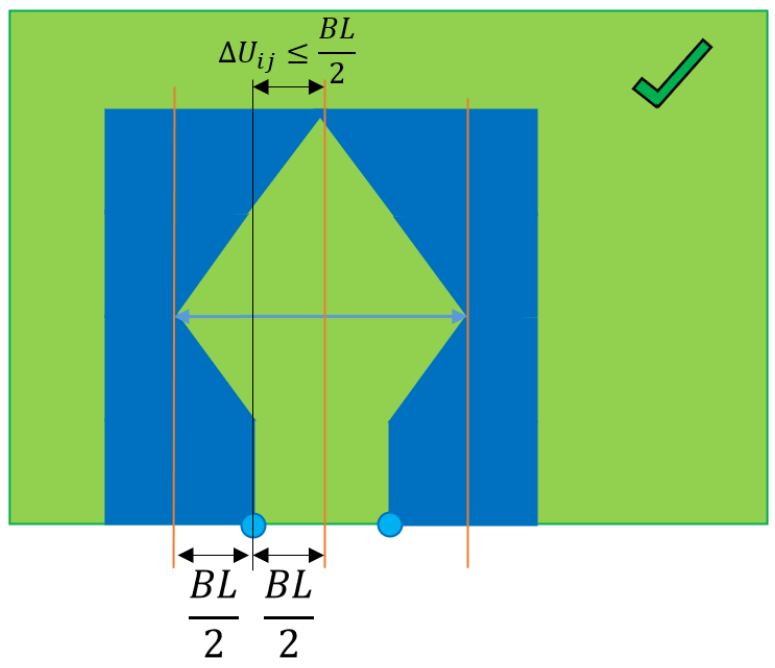

(b) Bridge length constraint satisfied

Fig. 6: Schematic representation of bridge length constraint. The solid line is the reference while the dashed line is the distance measured. Not shown in this representation is that the pointed tip is also under this constraint. the optimizer is overburdened by such a large number of constraints, especially where a finer resolution of curvature is required for actual design synthesis. One method presented is to use the $\operatorname{Max}()$ function, a commonplace function across any given programming language which has the disadvantage of non-existence of a derivative.

Therefore, a derivable aggregative and maximizing function is suggested to be used, and among many, the KreisselmeierSteinhauser (hereby KS) function [39], specifically, the lower bound KS function is employed that approximates the local overhang constraint violation maximum, given by:

$$
\begin{array}{r}
G_{K S}^{l}=C_{\max }+\frac{1}{P} \ln \left(\frac{1}{N_{C}} \sum_{i=1}^{N_{C}} e^{P\left(C_{i}-C_{\max }\right)}\right) \\
\forall i=1,2, \ldots . . N_{C}=2 n_{p}\left(n_{y}-1\right)
\end{array}
$$

Where $\mathrm{C}$ are the constraints, $\mathrm{P}$ is the hyperparameter of the KS function, $N_{C}$ is the total number of constraints and $C_{\max }$ is the maximum of the presented set of constraints derived using the $\operatorname{Max}()$ function. Further properties of this function are explained in detail in [39] and [15].

\subsection{Optimization Process}

The number of design variables is an important parameter to consider when choosing the optimizer. For significantly smaller number of variables of an Eulerian approach with similar application, a binary optimiser is most suited due to its ability to provide 0/1 solutions. For applications like Lagrangian approach based structural optimization, where large numbers of continu- 
ous variables are presented, a gradient based optimizer is most suited.

The most classical optimizer used for this purpose was the Optimality Criteria (OC) seen initially in its refined form in Top88 by Andreassen et al. [40] that used the bi-section method to find the optimal solution. For multiple constraints, the optimizer commonly used is the Method of Moving Asymptotes (MMA) [41], which has been implemented here.

The MMA is an iterative method, where the original problem is approximated by generating and solving a convex subproblem. A set of parameters that influence the curvature (convexity) of the approximations play an important role, and also act as the "asymptotes" for the generated subproblems. These asymptotes are moved between each iteration, thereby stabilizing the convergence of the overall solution. The complete list of all the hyper-parameters used for obtaining the results shown in Section 3 is shown in Table 2 .

\section{Results}

In order to demonstrate the discussed method, various example problems will be considered with different constraints enabled to show its effect on the final design solution. Solutions from traditional frameworks will also be presented to provide a reference solution. Reference solutions include SIMP, both with and without AM filter from [24], which is the latest opensource program available. Examples include the classic MBB beam (Fig. 77.top) and the cantilever beam (Fig. 77-bottom). The objective is to minimize compliance while adhering to maximum volume fraction constraint, whose mathematical formulation is given in $\mathrm{Eq} 24$

Many parameters are tuned such that the results remain comparable. A resolution of $100 \times 50$ is used for $\mathrm{MBB}$ beam and a resolution of $100 \times 100$ used for Short Cantilever. All cases have a maximum iteration limit of 4000 if no convergence is possible. Variations in the baseline inputs have been kept to a strict minimum to represent the methodology offered efficiently. The colorbar presented in any component plot refers to the mass of the components. A tabulated version of all the cases, its com-

\begin{tabular}{|c|c|c|}
\hline Parameter name & symbol| & value \\
\hline Material Young Modulus & $E$ & 1 \\
\hline $\begin{array}{l}\text { Design zone width (out of plane } \\
\text { direction) }\end{array}$ & $b$ & 1 \\
\hline Load amplitude & $F$ & 1 \\
\hline Poisson ratio & $v$ & 0.3 \\
\hline element size in $\mathrm{x}$ direction & $d x$ & 1 \\
\hline element size in y direction & $d y$ & 1 \\
\hline AMNA parameter $\gamma_{v}$ & $\gamma_{v}$ & 1 \\
\hline AMNA parameter $\gamma_{c}$ & $\gamma_{c}$ & 1 \\
\hline AMNA parameter $\varepsilon$ & $\varepsilon$ & 3 \\
\hline AMNA parameter $p_{b}$ & $p_{b}$ & 3 \\
\hline AMNA parameter $E_{\min }$ & $E_{\min }$ & $10^{-6}$ \\
\hline Aggregation constant & $\kappa$ & 10 \\
\hline MMA moving limit & & 0.01 \\
\hline MMA initial moving limit & & 0.01 \\
\hline MMA incremental factor asyincr & & 1.2 \\
\hline MMA decremental factor asydecr & & 0.4 \\
\hline MMA parameter albefa & & 0.1 \\
\hline $\begin{array}{c}\text { Stopping criterion, design variable } \\
\text { variation }\end{array}$ & & 0.001 \\
\hline No. of Components & $n_{p}$ & 6 \\
\hline No. of Layers per component & $n_{y}$ & 18 \\
\hline Maximum volume fraction & volfrac & 0.4 \\
\hline Minimal x position & $x_{\min }$ & $-\sqrt{n_{e l x}^{2}+n_{e l y}^{2}}$ \\
\hline Minimal length & $l_{\min }$ & 3 \\
\hline Minimal print height & $h_{\min }$ & 0 \\
\hline Minimal angle & $\theta_{\min }$ & $-2 \pi$ \\
\hline Minimal component density & $m_{\min }$ & 0 \\
\hline Maximal x position & $x_{\max }$ & $\sqrt{n_{e l x}^{2}+n_{e l y}^{2}}$ \\
\hline Maximal length & $l_{\max }$ & $n e l_{x} / n_{p}$ \\
\hline Maximal print height & $h_{\max }$ & $\sqrt{n_{e l x}^{2}+n_{e l y}^{2}}$ \\
\hline Maximal angle & $\theta_{\max }$ & $2 \pi$ \\
\hline Maximal component density & $m_{\max }$ & 1 \\
\hline
\end{tabular}

Table 2: Parameters used for the test cases

pliances and deviations, including its baseplate orientation is provided in Table 3

\subsection{Reference results}

Results from the basic implementation of SIMP and GGP (using only AMNA methodology) are provided in Fig. 11. The compliance values are provided to indicate the similarity of the final objective function, without any intent to state whether a given method is superior or inferior. The compliance of MBB Beam and Cantilever from the SIMP methodology is noted to be the least due to superior freedom in solution formation, while for the GGP-AMNA method it is 104.8 and 18.35 respectively.. 

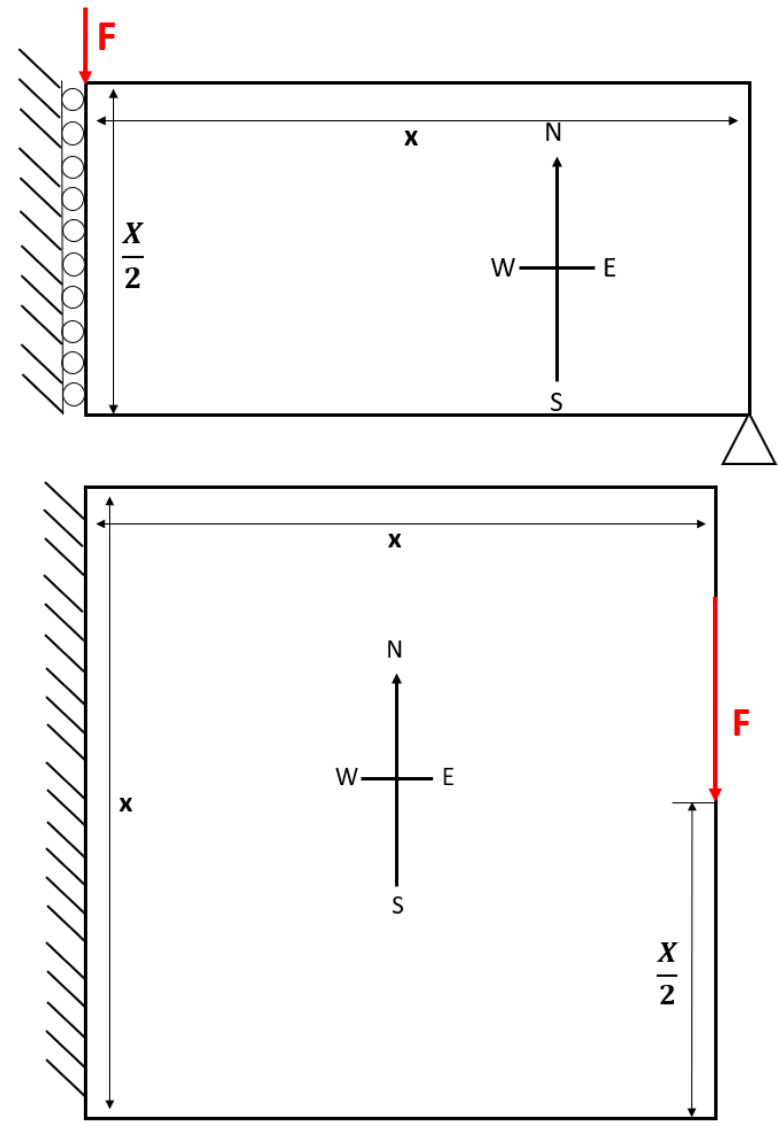

Fig. 7: Example problems considered: (top) The MBB Beam, (bottom) The short cantilever beam

Attention must be drawn to the near impracticality of these solutions for being additively manufactured without adding extensive support structures.

For the comparison of AM enabled solution using the GGP framework, solutions from the SIMP + AMfilter [24] have also been provided in Fig. 12, with compliance values of 106.6, $102.12,102.9,101.59$ and $19.43,18.58,19.44,18.47$ for MBB and Cantilever beam respectively (arranged in N, E, S, W respectively). In order to be critical of the presented framework, the lowest compliance values in each case and sub-case was considered as a baseline (and hence shown in Table 3) and the deviation provided will be with respect to these selected values, with positive values indicating that the compared value is higher than the SIMP + AM filter reference value. The baseplate orientation has been highlighted with a blue line.

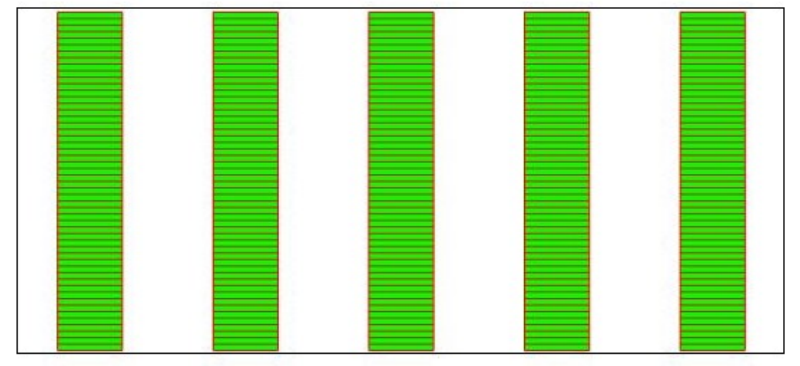

Fig. 8: A representation of initial component distribution using methodology from 36].

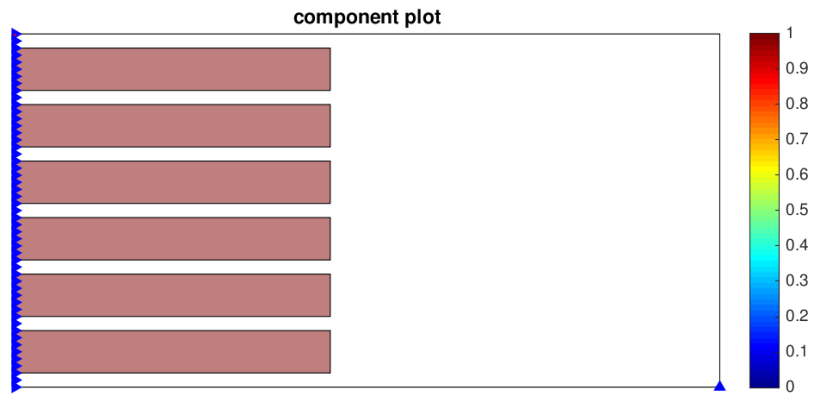

Fig. 9: A representation of initial component distribution in GGP - ALM framework.

\subsection{Results with no ALM constraints}

Results from the previous implementation of MNA in [36] has been quite encouraging, which was the main impetus for this framework where a band-like structure with multiple control points was envisioned for components. Unlike the current GGP framework, parameters were vague and control of these parameters were not straightforward, due to which there are rather obvious differences in the presented density and component plots. Some solutions for MBB and cantilever beam, whose components have a very large transition area is presented in Fig. 10, with the initial component distribution shown in Fig. 8

Using similar components in the presented framework, along with several other variables as discussed, has enabled solutions to support free 3D printing structures. The solutions with constraints will be presented in the latter sections of this article, while the solutions without 3D printing constraints have been presented in Fig. 13 and 14, with an initial component distribution shown in Fig. 9 for all cases hereafter. It can be observed that the extremely thin components, while visible in the component plot, is not reflected on the density plot. This is due to the 


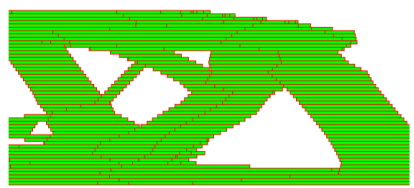

(a) MBB component plot

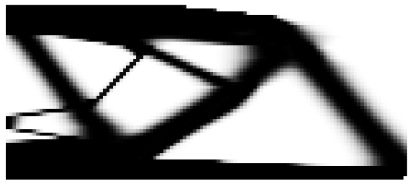

(b) MBB density plot

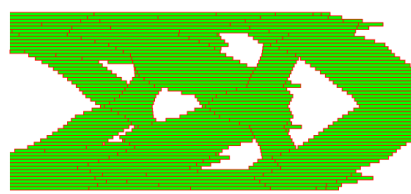

(c) Cantilever component plot

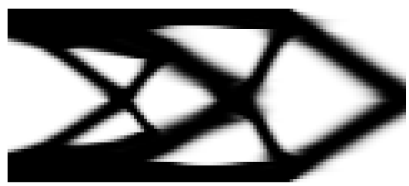

(d) Cantilever density plot

Fig. 10: Solution plots for 2 examples using methodology from [36]

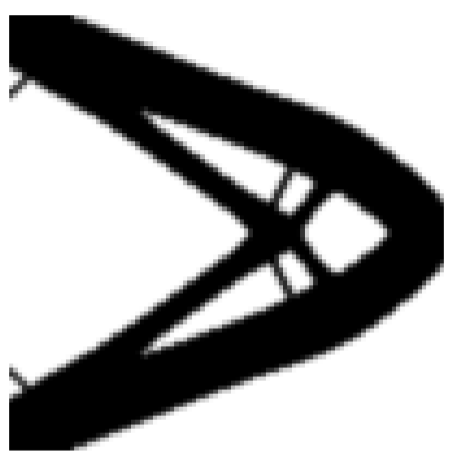

(a) Cantilever solution from SIMP

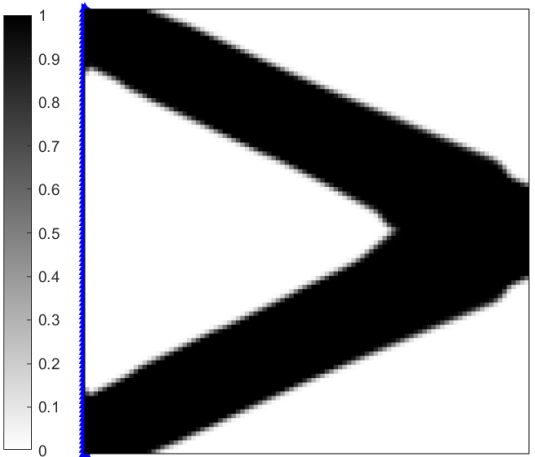

(b) Cantilever density plot from GGP

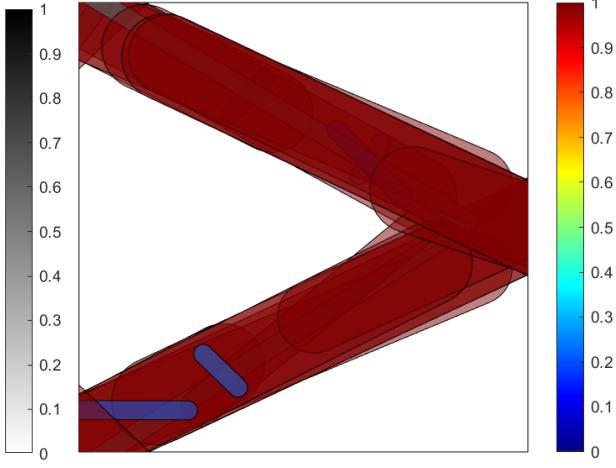

(c) Cantilever component plot from GGP

Fig. 11: Solution plots for cantilever beam using SIMP and GGP-AMNA

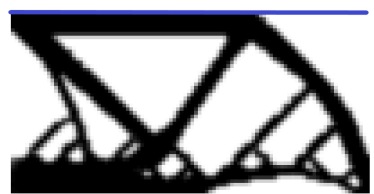

(a) Baseplate: North (106.6)

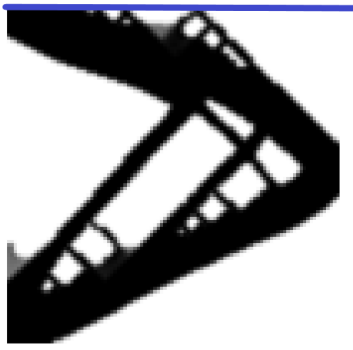

(e) Baseplate: North (19.43)

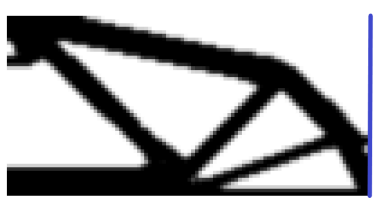

(b) Baseplate: East (102.12)

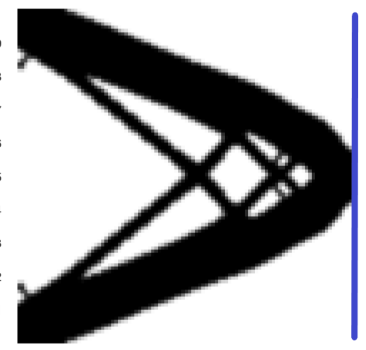

(f) Baseplate: East (18.58)

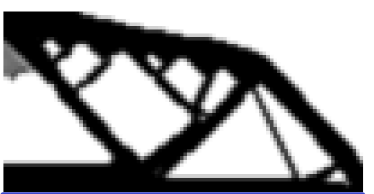

(c) Baseplate: South (102.9)

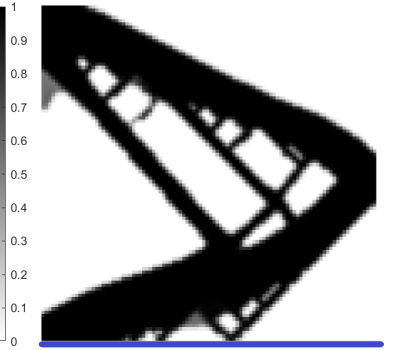

(g) Baseplate: South (19.44)

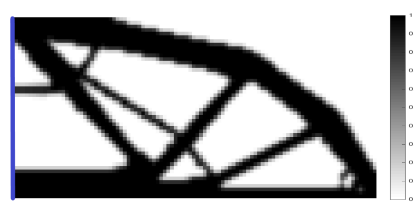

(d) Baseplate: West (101.59)

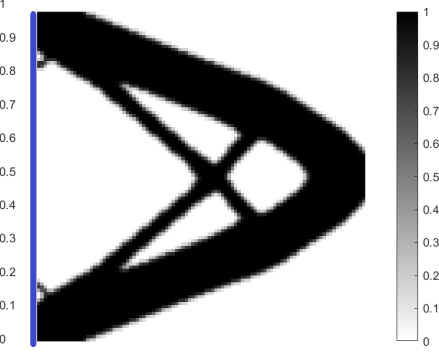

(h) Baseplate: West (18.47)

Fig. 12: Solution for two example problems (top: MBB beam and bottom: Cantilever beam) using the SIMP + AMfilter methodology. Values provided in parentheses are the compliance values of the corresponding case.

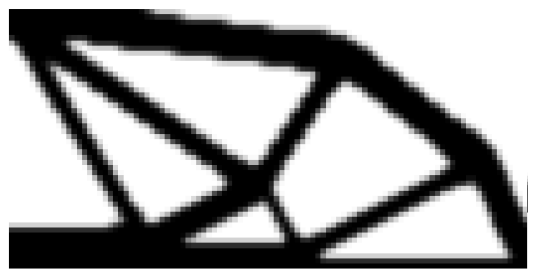

(a) SIMP density plot
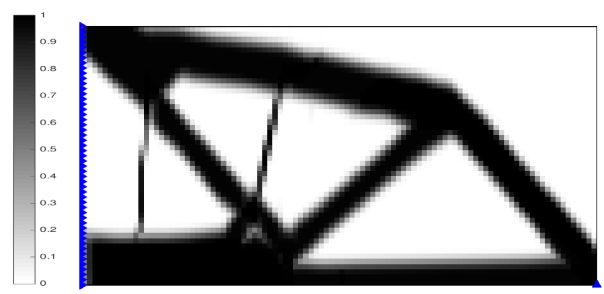

(b) GGP-AMNA density plot

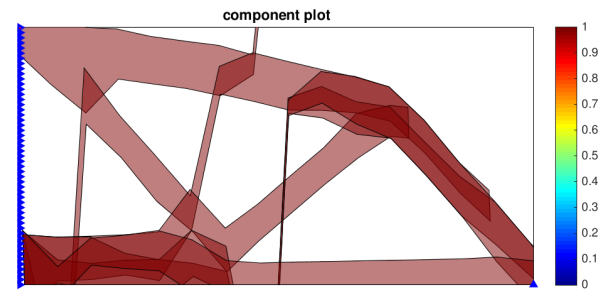

(c) GGP-AMNA component plot

Fig. 13: Solution plots for the MBB beam boundary condition without ALM constraints. Solutions from SIMP are provided for comparison. 


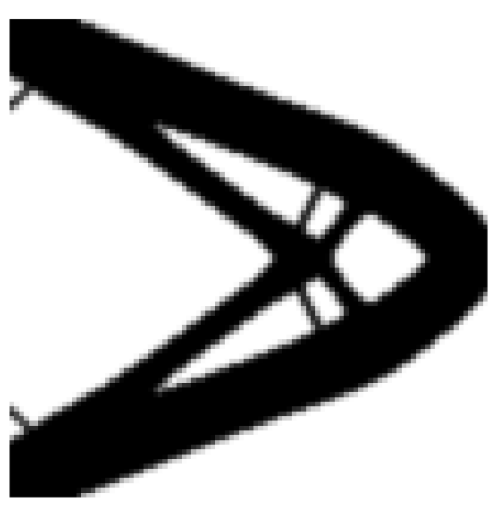

(a) SIMP density plot

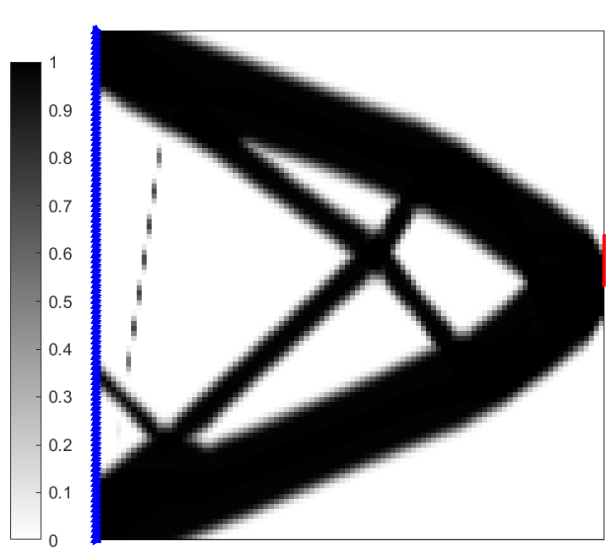

(b) GGP-AMNA density plot

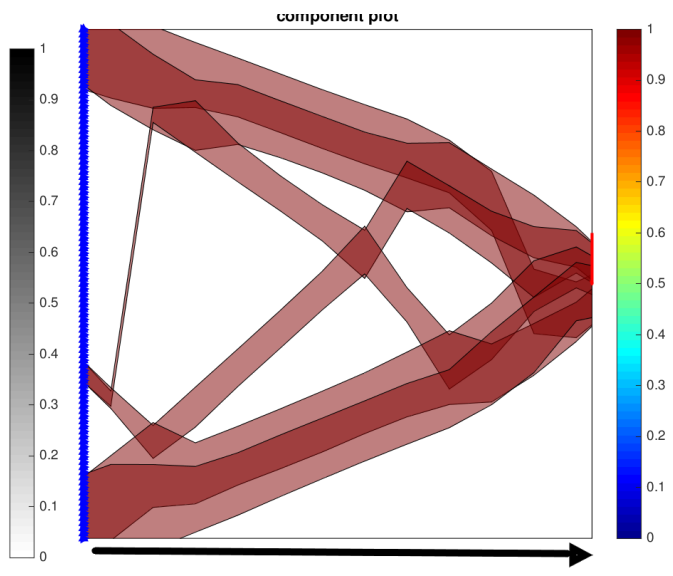

(c) GGP-AMNA component plot

Fig. 14: Solution plots for Short Cantilever boundary condition using GGP-AMNA methodology without ALM constraints. Solutions from SIMP are provided for comparison.

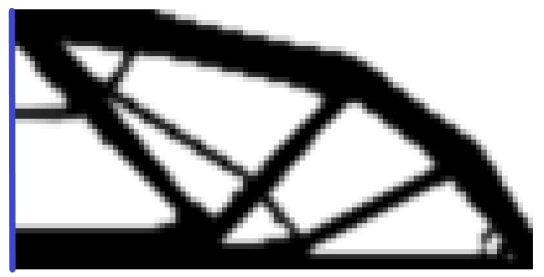

(a) SIMP + AMfilter with West baseplate density plot

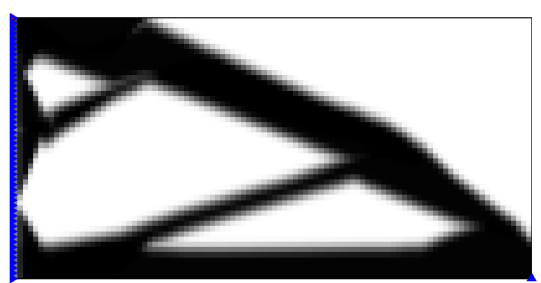

(b) MBB density plot

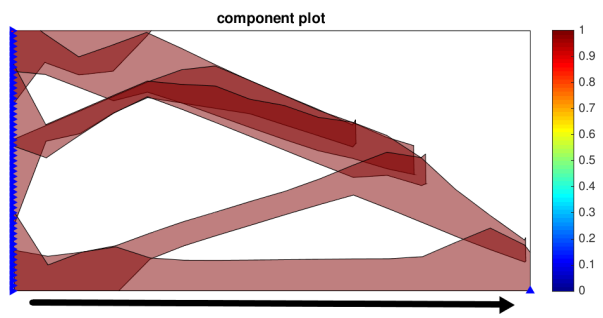

(c) MBB component plot

Fig. 15: Solution plots for MBB beam boundary condition using GGP-AMNA methodology with ALM constraints. Solution from SIMP + AM filter provided for comparison.

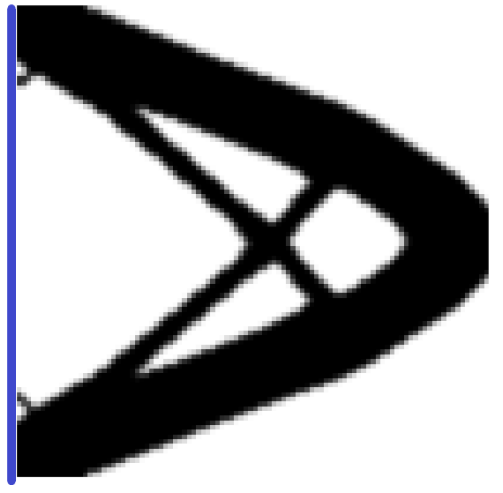

(a) SIMP + AMfilter with West baseplate density plot

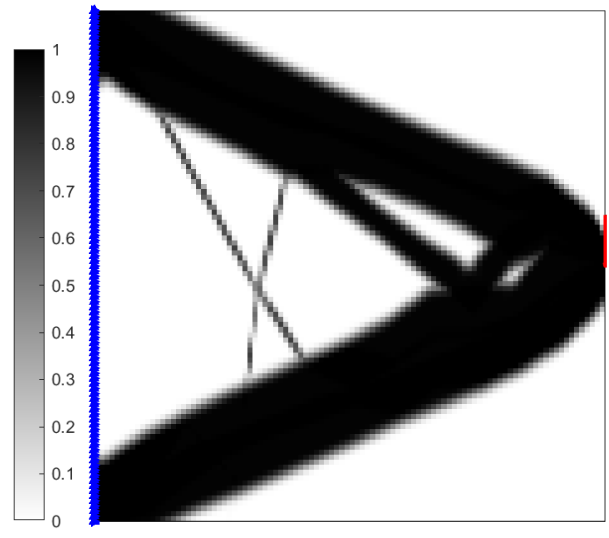

(b) Cantilever density plot

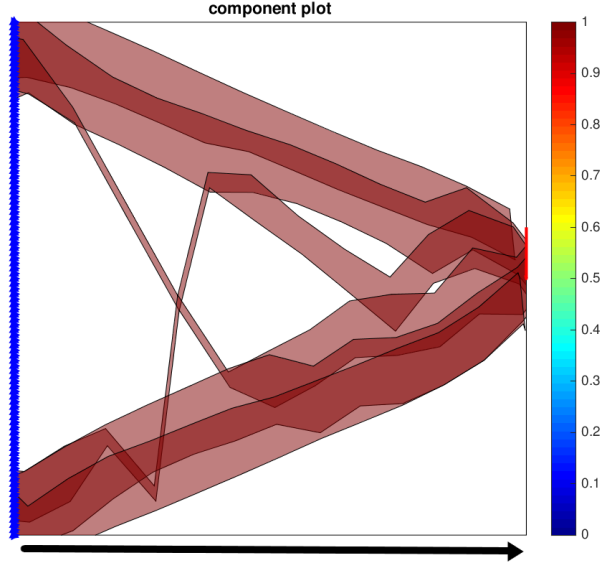

(c) Cantilever component plot

Fig. 16: Solution plots for Short Cantilever boundary condition using GGP-AMNA methodology with ALM constraints. Solution from SIMP + AM filter provided for comparison. 


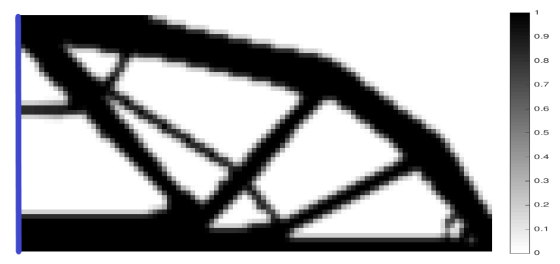

(a) SIMP + AMfilter with West baseplate density solution

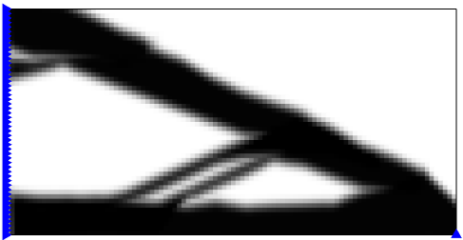

(b) MBB density solution with $\max \mathrm{BL}=20$

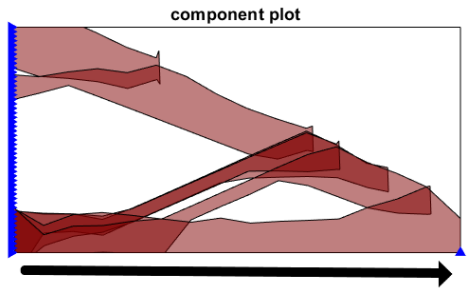

(e) MBB component solution with $\max \mathrm{BL}=20$

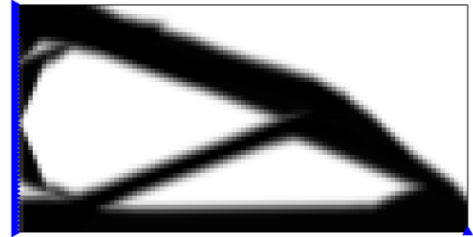

(c) $\mathrm{MBB}$ density solution with $\max \mathrm{BL}=25$

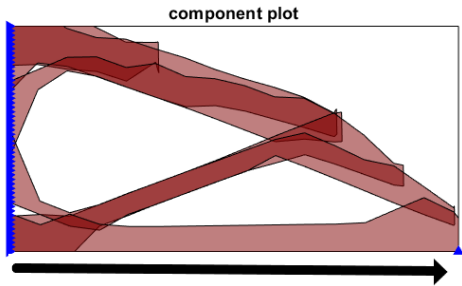

(f) MBB component solution with $\max \mathrm{BL}=25$

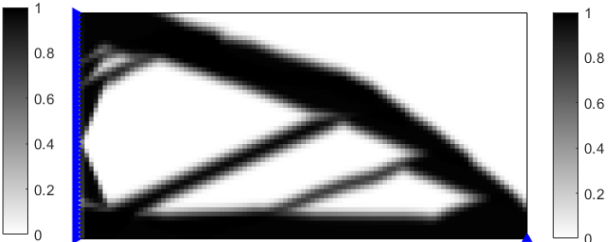

(d) MBB density solution with $\max \mathrm{BL}=30$

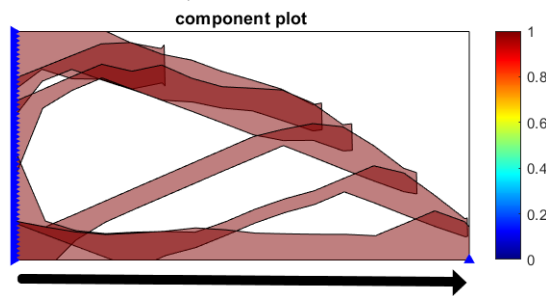

(g) MBB component solution with $\max \mathrm{BL}=30$

Fig. 17: Solutions for MBB beam with with set overhang angle and gradual relaxation of bridge length (BL) constraint.

\begin{tabular}{|c|c|c|c|c|c|}
\hline \multirow{2}{*}{ Method } & \multicolumn{2}{|c|}{ ALM Constraints } & \multirow{2}{*}{ Compliance } & \multirow{2}{*}{ Deviation } & \multirow{2}{*}{ Baseplate Orientation } \\
\hline & Overhang Angle & Bridge Length & & & \\
\hline \multicolumn{6}{|c|}{ MBB Beam } \\
\hline SIMP & $\mathrm{X}$ & $\mathrm{X}$ & 100.82 & 0 & $\mathrm{X}$ \\
\hline SIMP + AMfilter & $\checkmark$ & $\mathrm{X}$ & 101.59 & 0 & W \\
\hline $\begin{array}{c}\text { GGP-AMNA } \\
\text { (Original framework) }\end{array}$ & $\mathrm{X}$ & $\mathrm{X}$ & 104.8 & $3.95 \%$ & $\mathrm{X}$ \\
\hline \multirow{5}{*}{$\begin{array}{c}\text { GGP - AMNA } \\
\text { (Presented framework with } \\
\text { new geometric primitive) }\end{array}$} & & & 104.86 & $3.75 \%$ & $\mathrm{~W}$ \\
\hline & $\checkmark$ & $\mathrm{X}$ & 115.3 & $13.5 \%$ & $\mathrm{~W}$ \\
\hline & $\checkmark$ & 20 & 128.3 & $26.3 \%$ & $\mathrm{~W}$ \\
\hline & $\checkmark$ & 25 & 116.2 & $14.4 \%$ & $\mathrm{~W}$ \\
\hline & $\checkmark$ & 30 & 117.7 & $15.4 \%$ & $\mathrm{~W}$ \\
\hline \multicolumn{6}{|c|}{ Short Cantilever } \\
\hline SIMP & $\mathrm{X}$ & $\mathrm{X}$ & 18.48 & 0 & $\mathrm{X}$ \\
\hline SIMP + AMfilter & $\checkmark$ & & 18.47 & 0 & $\mathrm{~W}$ \\
\hline $\begin{array}{c}\text { GGP-AMNA } \\
\text { (Original framework) }\end{array}$ & $\mathrm{X}$ & $\mathrm{X}$ & 18.35 & $-0.7 \%$ & $\mathrm{X}$ \\
\hline \multirow{5}{*}{$\begin{array}{c}\text { GGP - AMNA } \\
\text { (Presented framework with } \\
\text { new geometric primitive) }\end{array}$} & $\mathrm{X}$ & $\mathrm{X}$ & 18.48 & $0 \%$ & $\mathrm{~W}$ \\
\hline & $\checkmark$ & $\mathrm{X}$ & 18.83 & $1.95 \%$ & $\mathrm{~W}$ \\
\hline & $\checkmark$ & 20 & 19.09 & $3.36 \%$ & W \\
\hline & $\checkmark$ & 25 & 19.51 & $5.6 \%$ & $\mathrm{~W}$ \\
\hline & $\checkmark$ & 30 & 19.24 & $4.2 \%$ & W \\
\hline
\end{tabular}

Table 3: Compliance, Deviation and Baseplate Orientation of design solutions obtained from reference and GGP+AMNA methods 

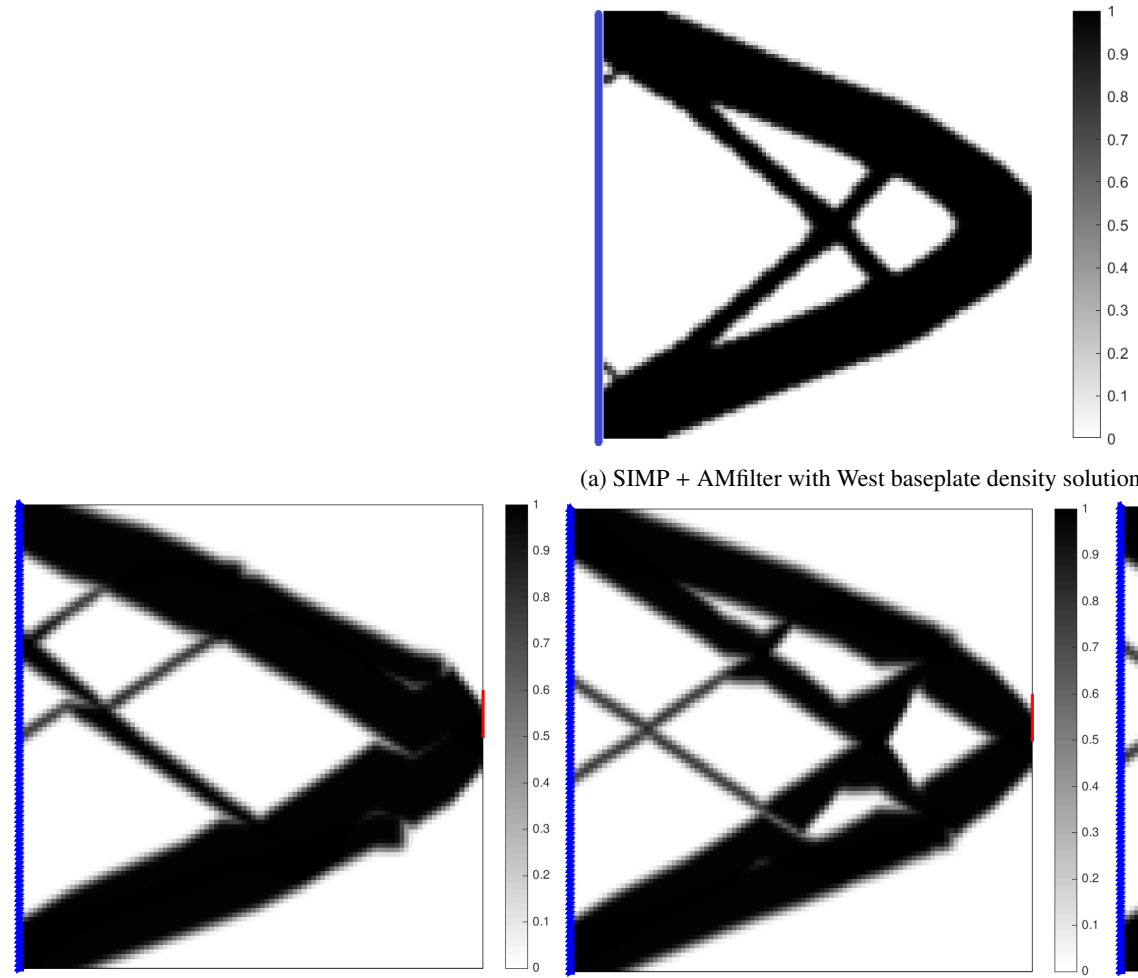

(a) SIMP + AMfilter with West baseplate density solution
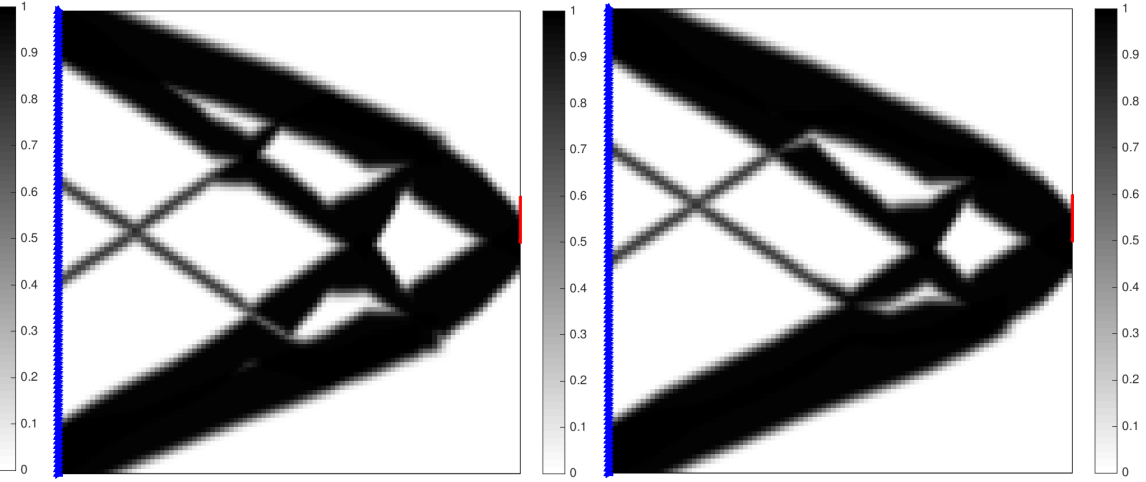

(b) Cantilever beam density solution with $\max B L=20$ (c) Cantilever beam density solution with max BL $=25$ (d) Cantilever beam density solution with max BL $=30$
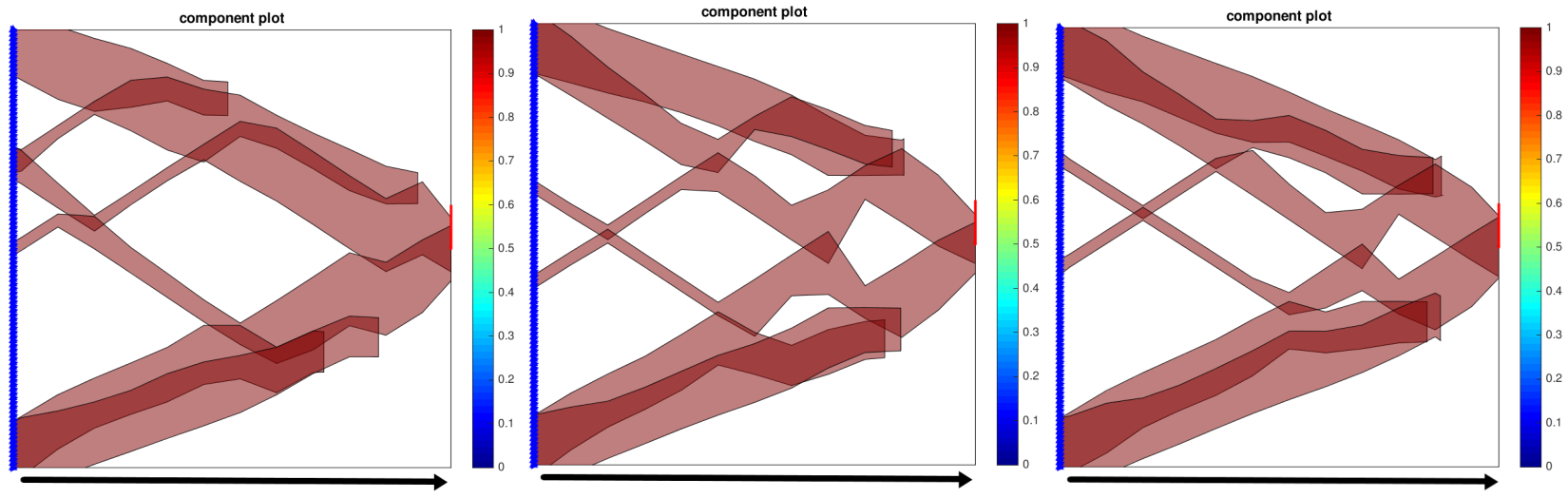

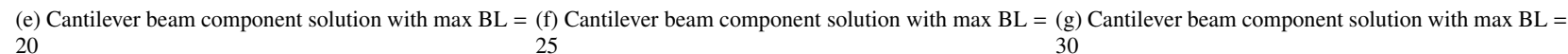

Fig. 18: Solutions for Cantilever beam with set overhang angle and gradual relaxation of bridge length (BL) constraint. 
hyperparameter for greyness transition distance being large, resulting in fine members being ignored on the density plot. One method to avoid thin members on the component plot is to increase the lower limit of component length $l_{\min }$.

\subsection{Results with only overhang angle constraint}

Having covered most of the reference solutions in the previous sub-sections, the solutions for AM enabled structures are presented in Fig. 15 and 16 Accompanying the solutions provided, the framework also provides the angle at which it can be printed and satisfy the constraints at the same time. The angles are continuous, unlike the strict baseplate orientation requirement observed in SIMP with AM filter. Since the optimum print direction is almost always corresponding to the west baseplate orientation (see Fig. 3 for reference), the optimum print angle shown in Table 3 reflects the same. The aggregation constant being too gentle, has obviously failed to strictly apply the overhang constraint for the Short Cantilever case, where the thin members seem to violate the overhang constraint.

\subsection{Results with overhang angle and bridge length constraints}

In this section, various bridge lengths are introduced gradually for constraints (with $\mathrm{BL}=20,25,30$ ), keeping the overhang angle constraint intact. Depending on the case, there are several trends to be observed. The results are provided in Fig. 17 for MBB beam and Fig. 18 for Cantilever beam.

The framework seems to provide practical solutions across the board. A decreasing trend is observed, where the objective values generally decrease as the BL constraint is increased due to the progressive relaxation of the component shape and position, effectively reducing the difference in optimum values, if not the final solution design. The minima also depends upon the symmetry of applied load with respect to the boundary condition, as evidenced in the case of MBB beam solution. This framework also demonstrates full control over projection parameters like the transition distance (i.e., the number of elements with values between 0 and 1 observed at the boundary of the projected component), as opposed to the arbitrary control previously implemented in [36]. As a final observation for the Short Cantilever case across all variations of constraints, it can be observed that the design solution is not symmetrical across the $\mathrm{X}$-axis, and therefore, the compliance can be considered as a local minima. Another trend observed during the simulation runs is the higher possibility of sub-optimal minima (i.e., local minima) if the initial component distribution contains too many components, thereby introducing higher number of thin components, resulting in higher greyness in the density map of the solutions.

\section{Conclusion}

Multi-disciplinary topology optimization frameworks are quite vital to enable additively manufactured solutions for critical components. Frameworks that support multiple requirements of the printing machine is a valid starting point for the same, proceeded by including material properties of the additively manufacturing material. The reference method of SIMP + AM filter has the highest degree of freedom and hence the objective values presented are the least for any provided cases, although it has the drawback of increased compute time in industrial applications, which makes geometric projection methods a necessity. The constraints are implemented using filters, and not explicitly as observed for projection methods like GGP.

Therefore, a framework whose computational time is independent from the fidelity of the problem being solved is required, and the presented GGP method demonstrates this ability. This particular framework has successfully mirrored the AM processes and has included geometric constraints using simple mathematical equations. The solutions thus obtained has been compared against established frameworks such as SIMP and SIMP with AMfilter. The optimum objective values obtained are compared strictly to provide only an overall analysis of the methods. This method provides a simpler gateway to study the thermal aspects of additive layer manufacturing without support inclusions. An effective study of thermal induced stresses and deformities is done by simulating layer-wise cyclic heating and cooling, which can effectively be represented by the presented framework. Another future work to be considered is to implement this framework using voxels in a 3D 
space, whose geometric primitive reflects the layer wise print-

ing of ALM machines and physically validate the same using commercially available ALM printers.

\section{Acknowledgments}

The authors would like to thank ISAE Fondation Class Gift Promo ' 83 for funding and enabling the Research Assistant position that lead this article to fruition.

\section{References}

[1] Wohlers, T, Gornet, T. History of additive manufacturing. Wohlers report 2014;24(2014):118.

[2] Gebhardt, A. Understanding additive manufacturing. Elsevier; 2011.

[3] Jiang, J, Xu, X, Stringer, J. Support structures for additive manufacturing: a review. Journal of Manufacturing and Materials Processing 2018;2(4):64.

[4] Fernandez-Vicente, M, Canyada, M, Conejero, A. Identifying limitations for design for manufacturing with desktop fff $3 \mathrm{~d}$ printers. International Journal of Rapid Manufacturing 2015;5(1):116-128.

[5] Bendsøe, MP, Kikuchi, N. Generating optimal topologies in structural design using a homogenization method. Computer methods in applied mechanics and engineering 1988;71(2):197-224.

[6] Bendsøe, MP, Sigmund, O. Material interpolation schemes in topology optimization. Archive of applied mechanics 1999;69(9-10):635-654.

[7] Zhou, M, Rozvany, G. The coc algorithm, part ii: Topological, geometrical and generalized shape optimization. Computer methods in applied mechanics and engineering 1991;89(1-3):309-336.

[8] Xie, YM, Steven, GP. A simple evolutionary procedure for structural optimization. Computers \& structures 1993;49(5):885-896.

[9] Wang, MY, Wang, X, Guo, D. A level set method for structural topology optimization. Computer methods in applied mechanics and engineering 2003;192(1-2):227-246.

[10] Allaire, G, Jouve, F, Toader, AM. Structural optimization using sensitivity analysis and a level-set method. Journal of computational physics 2004;194(1):363-393.

[11] Overvelde, JT. The moving node approach in topology optimization. Master's thesis; Technische Universiteit Delft; 2012.

[12] Norato, J, Bell, B, Tortorelli, D. A geometry projection method for continuum-based topology optimization with discrete elements. Computer Methods in Applied Mechanics and Engineering 2015;293:306327. doi $10.1016 / \mathrm{j}$.cma.2015.05.005

[13] Zhang, W, Yuan, J, Zhang, J, Guo, X. A new topology optimization approach based on moving morphable components (MMC) and the ersatz material model. Structural and Multidisciplinary Optimization 2015;53(6):1243-1260. doi 10.1007/s00158-015-1372-3

[14] Zhang, W, Yang, W, Zhou, J, Li, D, Guo, X. Structural topology optimization through explicit boundary evolution. Journal of Applied Mechanics 2016;84(1):011011. doi 10.1115/1.4034972

[15] Coniglio, S, Morlier, J, Gogu, C, Amargier, R. Generalized geometry projection: A unified approach for geometric feature based topology optimization. Archives of Computational Methods in Engineering 2019;:138.

[16] Zhou, M, Fleury, R, Shyy, YK, Thomas, H, Brennan, J. Progress in topology optimization with manufacturing constraints. In: 9th AIAA/ISSMO Symposium on multidisciplinary analysis and optimization. 2002, p. 5614.

[17] Zuo, KT, Chen, LP, Zhang, YQ, Yang, J. Manufacturing-and machining-based topology optimization. The international journal of advanced manufacturing technology 2006;27(5-6):531-536.

[18] Vatanabe, SL, Lippi, TN, de Lima, CR, Paulino, GH, Silva, EC. Topology optimization with manufacturing constraints: A unified projectionbased approach. Advances in Engineering Software 2016;100:97-112.
[19] Liu, J, Gaynor, AT, Chen, S, Kang, Z, Suresh, K, Takezawa, A, et al. Current and future trends in topology optimization for additive manufacturing. Structural and Multidisciplinary Optimization 2018;57(6):24572483.

[20] Plocher, J, Panesar, A. Review on design and structural optimisation in additive manufacturing: Towards next-generation lightweight structures. Materials \& Design 2019;:108164.

[21] Gaynor, AT, Guest, JK. Topology optimization considering overhang constraints: Eliminating sacrificial support material in additive manufacturing through design. Structural and Multidisciplinary Optimization 2016;54(5):1157-1172.

[22] Gaynor, AT, Meisel, NA, Williams, CB, Guest, JK. Topology optimization for additive manufacturing: considering maximum overhang constraint. In: 15th AIAA/ISSMO multidisciplinary analysis and optimization conference. 2014, p. 2036.

[23] Langelaar, M. Topology optimization of 3d self-supporting structures for additive manufacturing. Additive Manufacturing 2016;12:60-70.

[24] Langelaar, M. An additive manufacturing filter for topology optimization of print-ready designs. Structural and multidisciplinary optimization 2017;55(3):871-883.

[25] Thore, CJ, Grundström, HA, Torstenfelt, B, Klarbring, A. Penalty regulation of overhang in topology optimization for additive manufacturing. Structural and Multidisciplinary Optimization 2019;60(1):59-67.

[26] Qian, X. Undercut and overhang angle control in topology optimization: a density gradient based integral approach. International Journal for Numerical Methods in Engineering 2017;111(3):247-272.

[27] Leary, M, Merli, L, Torti, F, Mazur, M, Brandt, M. Optimal topology for additive manufacture: A method for enabling additive manufacture of support-free optimal structures. Materials \& Design 2014;63:678-690.

[28] Mirzendehdel, AM, Suresh, K. Support structure constrained topology optimization for additive manufacturing. Computer-Aided Design 2016;81:1-13.

[29] Zhang, W, Zhong, W, Guo, X. An explicit length scale control approach in simp-based topology optimization. Computer Methods in Applied Mechanics and Engineering 2014;282:71-86.

[30] Mass, Y, Amir, O. Topology optimization for additive manufacturing: Accounting for overhang limitations using a virtual skeleton. Additive Manufacturing 2017;18:58-73.

[31] Allaire, G, Dapogny, C, Estevez, R, Faure, A, Michailidis, G. Structural optimization under overhang constraints imposed by additive manufacturing technologies. Journal of Computational Physics 2017;351:295-328.

[32] Guo, X, Zhou, J, Zhang, W, Du, Z, Liu, C, Liu, Y. Self-supporting structure design in additive manufacturing through explicit topology optimization. Computer Methods in Applied Mechanics and Engineering 2017;323:27-63.

[33] Xian, Y, Rosen, DW. Morphable components topology optimization for additive manufacturing. Structural and Multidisciplinary Optimization $2020 ;: 1-21$.

[34] Langelaar, M. Integrated component-support topology optimization for additive manufacturing with post-machining. Rapid Prototyping Journal 2019;.

[35] Wein, F, Stingl, M. A combined parametric shape optimization and ersatz material approach. Structural and Multidisciplinary Optimization 2018;57(3):1297-1315.

[36] Bhat, KV, Coniglio, S, Morlier, J, Charlotte, M. Une approche par projection pour l'optimisation topologique de structures imprimées par fabrication additive. In: 14éme Colloque National en Calcul des Structures. 2019,.

[37] Norato, JA. A geometry projection method for the optimal distribution of short fiber reinforcements. In: International Design Engineering Technical Conferences and Computers and Information in Engineering Conference; vol. 57083. American Society of Mechanical Engineers; 2015, p. V02BT03A010.

[38] Zhang, S, Norato, JA, Gain, AL, Lyu, N. A geometry projection method for the topology optimization of plate structures. Structural and Multidisciplinary Optimization 2016;54(5):1173-1190.

[39] Kreisselmeier, G, Steinhauser, R. Systematic control design by optimizing a vector performance index. In: Computer aided design of control systems. Elsevier; 1980, p. 113-117.

[40] Andreassen, E, Clausen, A, Schevenels, M, Lazarov, BS, Sigmund, O. Efficient topology optimization in MATLAB using 88 lines of code. Structural and Multidisciplinary Optimization 2010;43(1):1-16. doi 10 . 
1007/s00158-010-0594-7

[41] Svanberg, K. The method of moving asymptotes-a new method for structural optimization. International Journal for Numerical Methods in Engineering 1987;24(2):359-373. doi 10.1002/nme.1620240207 